\title{
The Microscopic Spectral Density of the Dirac Operator derived from Gaussian Orthogonal and Symplectic Ensembles
}

\author{
Christian Hilmoine and Rune Niclasen \\ The Niels Bohr Institute \\ Blegdamsvej 17 \\ DK-2100 Copenhagen, Denmark
}

December 21, 2018

\begin{abstract}
The microscopic spectral correlations of the Dirac operator in Yang-Mills theories coupled to fermions in $(2+1)$ dimensions can be related to three universality classes of Random Matrix Theory. In the microscopic limit the Orthogonal Ensemble (OE) corresponds to a theory with 2 colors and fermions in the fundamental representation and the Symplectic Ensemble (SE) corresponds to an arbitrary number of colors and fermions in the adjoint representation. Using a new method of Widom, we derive an expression for the two scalar kernels which through quaternion determinants give all spectral correlation functions in the Gaussian Orthogonal Ensemble (GOE) and in the the Gaussian Symplectic Ensemble (GSE) with all fermion masses equal to zero. The result for the GOE is valid for an arbitrary number of fermions while for the GSE we have results for an even number of fermions.
\end{abstract}

NBI-HE-00-19

hep-th/0004081 


\section{Introduction}

Random Matrix Theory has successfully been used to extract information about the spectral correlations of the Euclidean Dirac operator $\not \supset=\gamma_{\mu}\left(\partial_{\mu}+i A_{\mu}\right)$ eigenvalues in the low energy limit of Yang-Mills theories such as QCD. In $(3+1)$ dimensions and in the low energy limit the effective YangMills partition function coincides with the partition function defined by the chiral Random Matrix Theory $(\chi \mathrm{RMT})$ when the so-called microscopic limit is taken 1, 2, 3, 那. The low-energy partition function describes the fermion mass dependence in the static limit and in a finite volume of space-time $V$ [5] and is determined alone by global symmetries. The finite volume implies that we restrict to the case where only the low-lying excitations (the Goldstone modes) contribute to the field theory partition function, while the kinetic terms of the Lagrangian are neglected. In the microscopic limit we look at the Dirac operator spectra on the scale $\lambda=\mathcal{O}\left(V^{-1}\right)$, which corresponds to a magnification of the spectra in the vicinity of $\lambda=0$ on the scale $V^{-1}$. In case of spontaneous chiral symmetry breaking, reflected in a condensate different from zero, $\Sigma \neq 0$, the scale $V^{-1}$ equals the average eigenvalue spacing. This follows from the Banks-Casher relation [6] $\Sigma=\pi \lim V^{-1} \rho(0)$, where $\rho(0)$ is the spectral density of the Dirac operator evaluated in origin and where first the thermodynamic and subsequently the chiral limit is taken. Thus we see that spontaneous chiral symmetry breaking is intimately related to the spectrum of the Dirac operator in origin.

In Random Matrix Theory no dynamical information is incorporated, only the global symmetries of the physical system are being used and therefore one studies the "universal" spectral correlations of the eigenvalues of the considered operator. Based on the work of Leutwyler and Smilga [7] it has been conjectured that the spectrum of the Dirac operator in QCD and similar theories is universal in the microscopic limit [1, 8]. This conjecture is supported by the fact that the sum rules derived by Leutwyler and Smilga, which involve inverse powers of the Dirac operator eigenvalues, can be derived from RMT [1, 2]. Thus, in this limit the microscopic spectral correlation functions (of which the microscopic spectral density is the most simple) of the Dirac operator can be derived from a much simpler Random Matrix Theory in which only the symmetries of the Dirac operator are the inputs. Depending on the representation of the gauge group $S U\left(N_{c}\right)$ and the number of colors $N_{c}$, the Dirac operator belongs to one of three universality classes, which in $\chi \mathrm{RMT}$ are represented by the orthogonal $(\chi \mathrm{OE})$, the unitary $(\chi \mathrm{UE})$ and the symplectic $(\chi \mathrm{SE})$ ensemble [9]. For each theory the symmetries of the Dirac operator specifies one of these universality classes. In addition the chiral structure of the Dirac operator in all three theories is incorporated in $\chi \mathrm{RMT}$, requiring a specific block structure of the matrices in the three ensembles $\chi \mathrm{UE}, \chi \mathrm{OE}$ and $\chi \mathrm{SE}$. See [2, 9] for a more detailed discussion.

Each of the three Yang-Mills theories in $(3+1)$ dimensions has an analogue in $(2+1)$ dimensions and the effective partition function of each theory has been showed to coincide with the partition function in each of the three universality classes defined by non- $\chi$ Random Matrix Theory (non- $\chi$ RMT) 41, 10, 11. In an odd number of space-time dimensions chiral symmetry does not exist. But in $(2+1)$ dimensions with an even number of flavors $N_{f}$ it has been suggested that the spontaneous breakdown of flavor symmetry occurs, and this is the analogue of spontaneous chiral symmetry breaking in $(3+1)$ dimensions [1, 12]. Thus, the argumentation of the entire picture in $(3+1)$ dimensions has a parallel in $(2+1)$ dimensions [1, 10, 11]. Here we learn again that an order parameter $\Sigma$ of the flavor symmetry breaking in $(2+1)$ dimensions is related to the spectral density of the Dirac operator, evaluated at zero, through a generalization of the Banks-Casher relation. The lack of chiral symmetry in $(2+1)$ dimensions is in the three ensembles non- $\chi \mathrm{OE}$, non- $\chi \mathrm{UE}$ and non- $\chi \mathrm{SE}$ reflected in the lack of the chiral block structure of the matrices. 
Thus in both $(3+1)$ and $(2+1)$ dimensions we have three types of Yang-Mills theories, defined by a choice of fermion colors $N_{c}$ and a representation of the gauge group $S U\left(N_{c}\right)$, and the symmetries of the Dirac operator in each theory implies a specific structure of the Dirac matrix. Each theory is represented by a random matrix ensemble: in $(3+1)$ dimensions we have the three chiral $(\chi)$ ensembles and in $(2+1)$ dimensions the three non-chiral (non- $\chi$ ) ensembles.

\begin{tabular}{||c|c|c|c|c||}
\hline \hline GAUGE GROUP & REPS. & DIRAC MATRIX & ENSEMBLE & $\beta$ \\
\hline \hline $\mathrm{SU}(2)$ & Fundamental & Real & $($ non- $) \chi \mathrm{OE}$ & 1 \\
\hline $\mathrm{SU}\left(N_{c} \geq 3\right)$ & Fundamental & Complex & $($ non- $) \chi \mathrm{UE}$ & 2 \\
\hline $\mathrm{SU}\left(N_{c}\right)$ & Adjoint & Quaternion real & $($ non- $) \chi \mathrm{SE}$ & 4 \\
\hline
\end{tabular}

Table 1: The table illustrates the classification, each ensemble is labelled by the Dyson index $\beta=1,2,4$.

In all three Yang-Mills theories in $(3+1)$ dimensions, as well as in $(2+1)$ dimensions we can have an arbitrary number of flavors $N_{f}$ of course. However, the coincidence of the ensembles $\beta=1$ and $\beta=4$ in $(2+1)$ dimensions with the corresponding effective field theory is only for even $N_{f}$. The relations to non- $\chi$ GOE and non- $\chi$ GSE of the two field theories was very recently derived by Magnea [10, 11]. In a theory with fermions in the fundamental representation of $S U(2)$ the following flavor symmetry breaking pattern causes the creation of the condensate $\Sigma[10]$ :

$$
S p\left(2 N_{f}\right) \rightarrow S p\left(N_{f}\right) \times S p\left(N_{f}\right) .
$$

In [11] the flavor symmetry breaking pattern

$$
O\left(N_{f}\right) \rightarrow O\left(N_{f} / 2\right) \times O\left(N_{f} / 2\right)
$$

is shown to be the one in a theory with $N_{c}$ arbitrary and the adjoint representation of $S U\left(N_{c}\right)$. In this paper we want to derive the massless microscopic spectral density of the Dirac operator in these two field theories from the Random Matrix Theory of non- $\chi \mathrm{OE}$ and non- $\chi \mathrm{SE}$.

The universality 13, 14 of the ensembles $\beta=1, \beta=2$ and $\beta=4$ allows the choice of a Gaussian distribution $(\mathrm{G})$ in these three ensembles, which is an advantage in view of the calculation of the spectral correlation functions. With the help of orthonormal polynomials all the microscopic spectral correlation functions have been derived in $\chi \mathrm{GOE}, \chi \mathrm{GUE}$ and $\chi \mathrm{GSE}$ with massive fermions, see review in 15 . In non- $\chi \mathrm{RMT}$, however, only the microscopic spectral correlation functions in non- $\chi \mathrm{UE}$ with an arbitrary number of massive fermions have been derived [4, 16, 17]. The two remaining universality classes are the non- $\chi \mathrm{OE}$ and non- $\chi \mathrm{SE}$. In this paper we derive the kernels $S_{N}^{(\beta)}(x, y)$ for non- $\chi \mathrm{GOE}$ and non- $\chi$ GSE with massless fermions. They determine all massless spectral correlation functions in these two ensembles. Specifically we derive the massless microscopic spectral density in the two ensembles. A direct verification of our results is possible through the generation of matrices distributed according to the probability distribution in the ensembles non- $\chi$ GOE and non- $\chi$ GSE. We also compare with the spectral sum rules recently derived in [10, 11].

The traditional method to derive spectral correlation functions in general orthogonal and symplectic ensembles with the use of polynomials is known as Dyson's quaternion matrix method [18]. In this method the kernels $S_{N}^{(\beta)}(x, y), \beta=1,4$, are represented by special sums involving skew-orthonormal polynomials and the spectral correlation functions are determined by the quaternion determinant of a quaternion matrix given by $S_{N}^{(\beta)}(x, y), \beta=1,4$. Widom and Tracy 24 have modified Dysons 
quaternion matrix method, in the sense that the polynomials in the relevant kernels now can be chosen arbitrary. In 19 the relevant kernels for the ensembles $\beta=1$ and $\beta=4$ are given by orthonormal polynomials. The main content of section 3 in this work is to provide a simple recipe for how to derive the two kernels in an ensemble defined by a general weight function, only by the use of orthonormal polynomials. We avoid the actual proof [19] and focus on the construction of a helpful machinery to derive the needed kernels. In section 4 we use the recipe on the non- $\chi$ Gaussian

ensembles $\beta=1,4$, defined in section 2 , and thus derive an expression for the two kernels $S_{N}^{(\beta)}(x, y)$. Although the equivalences between the field theory partition function and the the partition functions in the two cases $\beta=1,4$, only are valid for even $N_{f}$, and thus the result only has interest for even $N_{f}$ in Yang-Mills theory in $(2+1)$ dimensions [10, 11, the method gives us the spectral correlation functions also for odd $N_{f}$ in the ensemble $\beta=1$. In section 5 we present results for the microscopic spectral densities in the two ensembles and compare them with spectral sum rules and with Monte Carlo simulations done directly on random matrices.

\section{The non- $\chi$ Gaussian ensembles}

The non- $\chi$ random matrix model is defined by the partition function [4, 10, 11]

$$
\mathcal{Z}_{N_{f}}^{(\beta)}(\mathcal{M})=\int D T P_{N_{f}}^{(\beta)}(T)=\int D T \prod_{f=1}^{N_{f}} \operatorname{det}\left(i T+m_{f}\right) e^{-\frac{N \Sigma \beta}{4} \operatorname{Tr} \mathrm{V}\left(T^{2}\right)},
$$

where $T$ is from an ensemble of hermitian $N \times N$ matrices and the integration is taken over the Haar measure $D T$. The Dyson index $\beta$ has the value $\beta=1$ for the orthogonal ensemble (non- $\chi \mathrm{OE}$ ), $\beta=2$ for the unitary ensemble (non- $\chi \mathrm{UE}$ ) and $\beta=4$ for the symplectic ensemble (non- $\chi \mathrm{SE}$ ), which corresponds to real, complex and quaternion real matrix elements respectively. The matrix model is for a generic potential $V(T)$, but the basic assumption of universality justify the use of a Gaussian distribution, consistent with no additional input but the symmetries of the system. The condensate is $\Sigma \neq 0$, the parameter $N_{f}$ is restricted to integers and the diagonalized mass matrix $\mathcal{M}$ is having $N_{f}$ masses in the diagonal. The name "non- $\chi$ " is attached due to the inclusion of the determinant in (3), which makes the integrand not always positive and which makes these ensembles rather different from the usual orthogonal and symplectic ensembles near zero.

Putting all fermion masses to zero and deleting the determinant term $\left(N_{f}=0\right)$ makes the ensemble equivalent to the well known (classical) Gaussian ensemble and the replacement of the determinant with its absolute value (or restriction to even $N_{f}$ ) gives the generalized Gaussian ensemble [20]. The matrices in the chiral $(\chi)$ ensembles are rectangular in general and have a specific block structure, the former a result of the incorporation of the analogue of topological charge in the Random Matrix Theory and the latter corresponds to the choice of a representation of the Dirac matrix in a chiral basis. The lack of topological charge and chiral transformations in an odd number of space-time dimensions is in the non- $\chi$ RMT model (3) reflected in the quadratic matrices with no additional constraints, but the symmetries in each of the ensembles $\beta=1,2,4$. This is exactly what separates the non- $\chi$ ensemble from the $\chi$ ensemble.

By decomposition of the matrices $T$ one can easily transform to integration over the $N$ eigenvalues $\left\{\lambda_{k}\right\}$ of $T$. Choosing the Gaussian distribution then gives the partition function

$$
\mathcal{Z}_{N_{f}}^{(\beta)}(\mathcal{M})=\int_{-\infty}^{\infty} \prod_{k=1}^{N} d \lambda_{k} \prod_{f=1}^{N_{f} / 2}\left(\lambda_{k}^{2}+m_{f}^{2}\right) e^{-\frac{N \beta \Sigma}{4} \lambda_{k}^{2}}\left|\Delta\left(\left\{\lambda_{k}\right\}\right)\right|^{\beta}=\int_{-\infty}^{\infty} \prod_{k=1}^{N} d \lambda_{k} \omega_{N_{f}}^{(\beta)}\left(\lambda_{k}\right)\left|\Delta\left(\left\{\lambda_{k}\right\}\right)\right|^{\beta},
$$




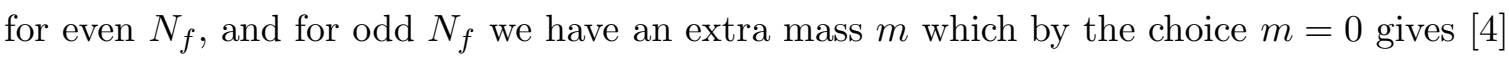

$\mathcal{Z}_{N_{f}}^{(\beta)}(\mathcal{M})=\int_{-\infty}^{\infty} \prod_{k=1}^{N} d \lambda_{k} \lambda_{k} \prod_{f=1}^{\left(N_{f}-1\right) / 2}\left(\lambda_{k}^{2}+m_{f}^{2}\right) e^{-\frac{N \beta \Sigma}{4} \lambda_{k}^{2}}\left|\Delta\left(\left\{\lambda_{k}\right\}\right)\right|^{\beta}=\int_{-\infty}^{\infty} \prod_{k=1}^{N} d \lambda_{k} \omega_{N_{f}}^{(\beta)}\left(\lambda_{k}\right)\left|\Delta\left(\left\{\lambda_{k}\right\}\right)\right|^{\beta}$,

were we have neglected all irrelevant overall factors. Here the function

$$
\Delta\left(\left\{\lambda_{i}\right\}\right) \equiv \prod_{i<j}^{N}\left(\lambda_{i}-\lambda_{j}\right)
$$

is the Vandermonde determinant and we have defined the weight function of the non- $\chi$ Gaussian ensemble

$$
\omega_{N_{f}}^{(\beta)}\left(\lambda_{k}\right) \equiv \begin{cases}\prod_{f=1}^{N_{f} / 2}\left(\lambda_{k}^{2}+m_{f}^{2}\right) e^{-\frac{N \beta \Sigma}{4} \lambda_{k}^{2}} & \text { for } N_{f} \text { even } \\ \lambda_{k} \prod_{f=1}^{\left(N_{f}-1\right) / 2}\left(\lambda_{k}^{2}+m_{f}^{2}\right) e^{-\frac{N \beta \Sigma}{4} \lambda_{k}^{2}} & \text { for } N_{f} \text { odd }\end{cases}
$$

on the support $\mathcal{I}=]-\infty, \infty[$. In the next section we outline the general method to derive the $m$-point spectral correlation function in a general ensemble defined by a weight function $\omega$. Note that, in the massless case, i.e all $m_{f}=0$, there is no separation between the two cases of even and odd $N_{f}$. Our goal is to derive an important function which determines the $m$-point spectral correlation functions in the two cases $\beta=1,4$ of the massless non- $\chi$ Gaussian ensembles.

\section{The $m$-point correlation function}

The spectrum of the ensemble defined by the general weight function $\omega$ on the support $\mathcal{I}$ is described by the m-point correlation function

$$
R_{m}^{(\beta)}\left(x_{1}, \ldots, x_{m}\right)=\frac{1}{\mathcal{Z}_{N_{f}}^{(\beta)}} \frac{N !}{(N-m) !} \int_{\mathcal{I}} d x_{m+1} \ldots d x_{N} \prod_{j=1}^{N} \omega\left(x_{j}\right)\left|\Delta\left(\left\{x_{i}\right\}\right)\right|^{\beta} .
$$

This function gives the probability density that $m$ of the eigenvalues, irrespective of their ordering, are located in infinitesimal neighborhoods of $x_{1}, \ldots, x_{m}$.

Now we will review how to derive the function $R_{m}^{(\beta)}$ with the help of orthonormal polynomials in all three ensembles $\beta=1,2,4$. Define the functions

$$
\varphi_{i}(x) \equiv p_{i}(x) \omega(x)^{1 / 2}, \quad i=0,1,2, \ldots,
$$

where $\left\{p_{i}(x)\right\}$ is the sequence of polynomials orthonormal with respect to the weight function $\omega(x)$ on $\mathcal{I}$. Thus the sequence $\left\{\varphi_{i}(x)\right\}$ consists of orthonormal functions on $\mathcal{I}$. Define the Hilbert space $\mathcal{H}$ spanned by the functions $\varphi_{i}(x), i=0,1,2, \ldots,(N-1)$. The projection operator $K$ onto the space $\mathcal{H}$ has the kernel

$$
K_{N}(x, y) \equiv \sum_{k=0}^{N-1} \varphi_{k}(x) \varphi_{k}(y)=\frac{a_{N}}{x-y}\left(\varphi_{N}(x) \quad \varphi_{N-1}(x)\right)\left(\begin{array}{cc}
0 & 1 \\
1 & 0
\end{array}\right)\left(\begin{array}{c}
\varphi_{N}(y) \\
\varphi_{N-1}(y)
\end{array}\right)
$$


where the last equality follows from the important Christoffel-Darboux (see [21]) formula and $a_{N}=$ $k_{N-1} / k_{N}, k_{N}$ denoting the highest coefficient in $p_{N}(x)$. In the unitary ensemble $(\beta=2)$ the use of orthonormality and a simple rewriting of $\left|\Delta\left(\left\{\lambda_{i}\right\}\right)\right|^{2}$ gives 18]

$$
R_{m}^{(2)}\left(x_{1}, \ldots, x_{m}\right)=\operatorname{det}\left[K_{N}\left(x_{i}, x_{j}\right)\right]_{1 \leq i, j \leq m} .
$$

In the orthogonal and symplectic ensembles we have an analogous result for $R_{m}^{(\beta)}, \beta=1,4$. Here, however, the corresponding kernel $K_{N}^{(\beta)}$ is not a scalar, but a quaternion kernel and $R_{m}^{(\beta)}$ is represented by the quaternion determinant of quaternion matrix $\left[K_{N}\left(x_{i}, y_{j}\right)\right]_{1 \leq i, j \leq m}$ [18]. Now, representing the quaternion kernels by their $2 \times 2$ matrix representations, whose entries all are given by one specific scalar kernel $S_{N}^{(\beta)}$, completes the analogous picture for the ensembles $\beta=1,4$ : The matrix kernels

$$
K_{N}^{(4)}(x, y)=\frac{1}{2}\left(\begin{array}{cc}
S_{N}^{(4)}(x, y) & S^{(4)} D(x, y) \\
I S_{N}^{(4)}(x, y) & S_{N}^{(4)}(y, x)
\end{array}\right),
$$

and

$$
K_{N}^{(1)}(x, y)=\left(\begin{array}{cc}
S_{N}^{(1)}(x, y) & S^{(1)} D(x, y) \\
I S_{N}^{(1)}(x, y)-\varepsilon(x-y) & S_{N}^{(1)}(y, x)
\end{array}\right),
$$

determine the $m$-point correlation function

$$
R_{m}^{(\beta)}\left(x_{1}, \ldots, x_{m}\right)=\mathrm{Q} \operatorname{det}\left[K_{N}^{(\beta)}\left(x_{i}, x_{j}\right)\right]_{1 \leq i, j \leq m}, \quad \beta=1,4 .
$$

Thus, the $m$-point correlation function is represented by the quaternion determinant of a $2 m \times 2 m$ matrix. Here $\varepsilon(x)=x /(2|x|)$ (the kernel of the operator $\varepsilon$ ), the scalar kernel $S_{N}^{(\beta)}(x, y)$ is given by certain sums of products involving the functions $\varphi_{n}$, and $I$ and $D$ are integration and differentiation operators, respectively. The operator $S^{(\beta)}$ has kernel $S_{N}^{(\beta)}(x, y), S^{(\beta)} D(x, y)$ is the kernel of the operator $S^{(\beta)} D$ and $I S_{N}^{(\beta)}(x, y)$ is the kernel of $I S_{N}^{(\beta)}$. We see that the entire matrix kernel $K_{N}^{(\beta)}(x, y)$ is given by the scalar kernel $S_{N}^{(\beta)}(x, y), \beta=1,4$. In the classical way derived by Dyson [22], and later generalized by Mehta and Mahoux [23], the polynomials in the functions $\varphi_{n}$, and therefore in $S_{N}^{(\beta)}(x, y)$, are chosen skew-orthonormal with respect to $\omega$, which through an important theorem by Dyson gives the result (14). In [24], it has been shown that any choice of a family of polynomials leads to the same matrix kernel $K_{N}^{(\beta)}(x, y), \beta=1,4$. The choice of skew-orthonormal polynomials leads apparently to the most simple $K_{N}^{(\beta)}(x, y)$. But the tedious work to derive skew-orthonormal polynomials and the lack of an relevant formula for this analogue of the Christoffel-Darboux formula (which is preferable when the scaling limit $N \rightarrow \infty$ is taken) asks for a representation of $S_{N}^{(\beta)}(x, y)$ in which the polynomials have well known properties and the sums involving them are more easy to deal with. This is precisely the result of [19]. For a weight function $\omega$, with respect to which there exist orthonormal polynomials, and for which the function $\omega^{\prime} / \omega$ is a rational function, the two kernels $S_{N}^{(\beta)}(x, y), \beta=1,4$, are given by 19 :

$$
\begin{gathered}
S_{N}^{(4)}(x, y)=K_{2 N}(x, y)-\sum_{i>n, j=1}^{2 n}\left[A_{0} C_{00}^{-1} C_{0}\right]_{i j} \psi_{i}(x) \varepsilon \psi_{j}(y), \\
S_{N}^{(1)}(x, y)=K_{N}(x, y)-\sum_{i \leq n, j=1}^{2 n}\left[A C(I-B A C)^{-1}\right]_{j i} \psi_{i}(x) \varepsilon \psi_{j}(y) .
\end{gathered}
$$


Both scalar kernels $S_{N}^{(\beta)}(x, y), \beta=1$, , equal a modified version of the scalar kernel (10) from the corresponding unitary ensemble, plus a linear combination of $n$ functions. The number $n$ equals the sum of orders of the poles of $\omega^{\prime} / \omega$ in the extended complex plane, where every endpoint of the support $\mathcal{I}$, where $\omega^{\prime} / \omega$ is analytic, is included as a simple pole. Thus, for the Gaussian ensemble $\left(\omega(x)=e^{-x^{2}}, \mathcal{I}=\right]-\infty, \infty[)$ we have $n=1$ because of the simple pole in $\infty$ and for the Legendre ensemble $(\omega(x)=1, \mathcal{I}=[-1,1]) n=2$ because of the two endpoints. For the weight function $\omega$ the polynomials in (16) (the ensemble $\beta=1$ ) are orthonormal with respect to $\omega^{2}$, meaning we must make the shift $\omega^{1 / 2} \rightarrow \omega$ in (9). In the function (15), the involved polynomials are orthonormal with respect to $\omega$, as in the ensemble with $\beta=2$, but here the shift $N \rightarrow 2 N$ must be taken. The relevant Hilbert space for each ensemble is thus

$$
\begin{array}{ll}
\beta=1: & \mathcal{H}=\operatorname{span}\left\{\varphi_{0}, \varphi_{1}, \ldots, \varphi_{N-1}\right\}, \quad \text { where } \quad \varphi_{i}(x)=p_{i}(x) \omega(x), \\
\beta=4: & \mathcal{H}=\operatorname{span}\left\{\varphi_{0}, \varphi_{1}, \ldots, \varphi_{2 N-1}\right\}, \quad \text { where } \quad \varphi_{i}(x)=p_{i}(x) \omega(x)^{1 / 2},
\end{array}
$$

and $p_{i}(x)$ is a polynomial of degree $i$. The functions $\psi_{i}(x), i=1,2, \ldots, 2 n$, are determined from the two functions $\varphi_{N}(x), \varphi_{N-1}(x)\left(N \rightarrow 2 N\right.$ for $\beta=4$ and $\omega^{1 / 2} \rightarrow \omega$ for $\left.\beta=1\right)$, and the poles of $\omega^{\prime} / \omega$. Once we have determined the $2 n$ linear independent functions, that is precisely $\psi_{i} \in \mathcal{H}, i=1,2, \ldots, n$, and $\psi_{i} \in \mathcal{H}^{\perp}, i=(n+1), \ldots, 2 n$, the matrices $A, B$ and $C$ (and $A_{0}, C_{00}$ and $C_{0}$, which just are block parts of $A$ and $C$ ) can be derived from these. In the next section we describe the procedure in great detail.

The results (15) and (16) have their origin in identities for each operator $S^{(\beta)}, \beta=1$, 4 , which are valid for a general $\omega$, telling that $S^{(\beta)}$ equals the projection operator $K$ onto the Hilbert space $\mathcal{H}$, plus a correction. This correction is in each ensemble $\beta=1,4$ given by the operators $K, D$, and $\varepsilon$, and in case the operator $[D, K]$ has finite rank, i.e independent of $N$, the kernel of this correction constitute finitely many terms. When $\omega^{\prime} / \omega$ is a rational function this is precisely the case, and the choice of orthonormal polynomials with respect to $\omega$ and $\omega^{2}$ for $\beta=4$ and $\beta=1$ respectively, leads to (15) and (16).

In the proceeding section we construct a recipe for how to derive the $n$ corrections on the right hand sides of (15) and (16).

\subsection{Deriving the kernels $S_{N}^{(\beta)}\left(x_{i}, x_{j}\right), \beta=1,4$, with the help of orthonormal polyno- mials}

For a semi-classical weight function $\omega$, i.e $\omega^{\prime} / \omega$ is a rational function, the following procedure [19] leads to the derivation of the two associated kernels $S_{N}^{(\beta)}\left(x_{i}, x_{j}\right), \beta=1,4$. We restrict to weight functions on the form

$$
\omega(x)=e^{-V(x)},
$$

fulfilling

$$
\lim _{x \rightarrow \partial \mathcal{I}} \omega(x)=0,
$$

where $V(x)$ is continuously differentiable in the interior of $\mathcal{I}$. (For certain weight functions the condition (19) is unnecessary [26].) The $n$ correction terms in each of the two ensembles are determined by the poles of $\omega^{\prime} / \omega$ and the two functions

$$
\begin{aligned}
\beta=1: & \varphi_{i}(x)=p_{i}(x) \omega(x), \quad i=(N-1), N \\
\beta=4: & \varphi_{i}(x)=p_{i}(x) \omega(x)^{\frac{1}{2}}, \quad i=(2 N-1), 2 N,
\end{aligned}
$$


where the polynomials $p_{i}$ are chosen orthonormal with respect to $\omega^{2}(x)=e^{-2 V(x)}$ and $\omega(x)=e^{-V(x)}$, respectively. The following steps give the right hand side of (15) and (16), where the specific functions given above, belonging to each ensemble, are used. In the ensemble $\beta=1$ we assume that $N$ is even. With the purpose to work with both ensembles at the same time we make the shift $2 N \rightarrow N$ for $\beta=4$. Therefore, in the end of the procedure we must remember to double up the matrix dimension $N \rightarrow 2 N$ for $\beta=4$. Here is the recipe summarizing the general method 19 for obtaining the two kernels $S_{N}^{(\beta)}(x, y), \beta=1,4$ :

(1) At first the main contribution to $S_{N}^{(\beta)}(x, y), \beta=1,4$, is determined. For each ensemble $\beta=1,4$, the kernel $K_{N}(x, y)$ is constructed from the associated two functions, (20) or (21), through (10).

(2) Using the definition

$$
U(x, y)=c^{(\beta)} \frac{V^{\prime}(x)-V^{\prime}(y)}{x-y}, \quad c^{(\beta)}= \begin{cases}1 & \text { for } \beta=4 \\ 2 & \text { for } \beta=1,\end{cases}
$$

calculate the four functions

$$
\begin{gathered}
A_{N}(x) \equiv a_{N} \int_{\mathcal{I}} d y \varphi_{N}(x) \varphi_{N-1}(y) U(x, y), \\
B_{N}(x) \equiv a_{N} \int_{\mathcal{I}} d y \varphi_{N}^{2}(y) U(x, y), \\
A(x) \equiv A_{N}(x)-\frac{c^{(\beta)}}{2} V^{\prime}(x), \quad C(x) \equiv \frac{a_{N}}{a_{N-1}} B_{N-1}(x),
\end{gathered}
$$

for each $\beta=1$, 4 . (The $c^{(\beta=1)}=2$ comes from the factor 2 in the exponent of the weight function

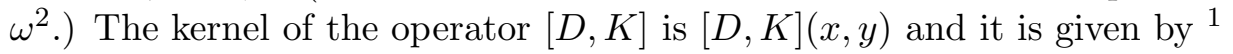

$$
[D, K](x, y)=a_{N}\left(\varphi_{N}(x) \quad \varphi_{N-1}(x)\right)\left(\begin{array}{cc}
\frac{C(x)-C(y)}{x-y} & \frac{A(x)-A(y)}{x-y} \\
\frac{A(x)-A(y)}{x-y} & \frac{B_{N}(x)-B_{N}(y)}{x-y}
\end{array}\right)\left(\begin{array}{c}
\varphi_{N}(y) \\
\varphi_{N-1}(y)
\end{array}\right) .
$$

Here the central matrix is vital for the derivation of the specific matrix $A$ (not to be confused with the function $A(x)$ ) later on.

(3) Next, the number $n=n_{\infty}+\sum_{j=1}^{N_{p}} n_{x_{j}}$ is calculated. The orders $\left\{n_{x_{j}}\right\}$ of the $N_{p}$ poles $\left\{x_{j}\right\}$ of the function $\omega^{\prime} / \omega$ are added. A pole at infinity with order $n_{\infty}$ is included as well as the endpoints of $\mathcal{I}$ in which $\omega$ is analytic, the latter are counted as simple poles $\left(n_{x_{j}}=1\right)$. (Notice that $\left(\omega^{2}\right)^{\prime} / \omega^{2}$, relevant for $\beta=1$, has the same poles with same orders as $\omega^{\prime} / \omega$.)

(4) By writing out the power series of the rational functions $A(x), B_{N}(x)$ and $C(x)$ in (26) one can easily verify that the kernel $[D, K](x, y)$ can be represented by a linear combination of the $2 n$ functions

$$
x^{k} \varphi_{N-1}(x), \quad x^{k} \varphi_{N}(x), \quad\left(0 \leq k<n_{\infty}\right),
$$

${ }^{1}$ This is easily derived with the help of [26]:

$$
\left(\begin{array}{c}
\varphi_{N}^{\prime}(x) \\
\varphi_{N-1}^{\prime}(x)
\end{array}\right)=\left(\begin{array}{cc}
A(x) & B_{N}(x) \\
-C(x) & -A(x)
\end{array}\right)\left(\begin{array}{c}
\varphi_{N}(x) \\
\varphi_{N-1}(x)
\end{array}\right),
$$

and the representation (10) of $K_{N}(x, y)$. 


$$
\left(x-x_{i}\right)^{-k-1} \varphi_{N-1}(x), \quad\left(x-x_{i}\right)^{-k-1} \varphi_{N}(x), \quad\left(0 \leq k<n_{x_{i}}\right) .
$$

In addition, the space, $\mathcal{G}$, spanned by these $2 n$ functions has a subspace of dimension $n$ contained in $\mathcal{H}$ and another subspace of dimension $n$ contained in $\mathcal{H}^{\perp}$. Therefore, we now determine $2 n$ linearly independent functions $\psi_{i}(x) \in \mathcal{H}, i=1,2, \ldots, n$, and $\psi_{i}(x) \in \mathcal{H}^{\perp}, i=(n+1), \ldots, 2 n$. It is easily shown that all the functions in $\mathcal{G}$ are orthogonal to all functions contained in the space $\mathcal{H} / \mathcal{S}$, where

$$
\mathcal{S}=\operatorname{span}\left\{\varphi_{N-k}, \varphi_{l}\right\}, \quad 0<k \leq n_{\infty}, \quad 0 \leq k<\left(n-n_{\infty}\right)
$$

is a subspace of $\mathcal{H}$ and has dimension $n$. This implies that the $n$ functions $\psi_{i} \in \mathcal{H}^{\perp}$ are given by the demand $\psi_{i} \in \mathcal{S}^{\perp}$, simplifying the derivation of the functions.

We have

$$
[D, K](x, y)=\sum_{i, j=1}^{2 n} A_{i j} \psi_{i}(x) \psi_{j}(y),
$$

where the $2 n \times 2 n$ matrix $A$ now must be derived through (26) by the specific choice of the functions $\psi_{i}(x), i=1,2, \ldots, 2 n$. The matrix $A$ is symmetric (because $K$ is symmetric and $D$ is anti-symmetric) and satisfies

$$
A_{i j}=0 \quad \text { if } \quad i, j \leq n \quad \text { or } \quad i, j>n,
$$

which reduces the work. There are not other restrictions on the choice of the functions $\psi_{i}$, but the linear independence, and the matrix $A$ is uniquely determined through (26) by the given choice.

(5) The $2 n \times 2 n$ matrix $B$ defined by the inner product

$$
B_{i j} \equiv\left(\varepsilon \psi_{i}, \psi_{j}\right)=\left(\int_{\mathcal{I}} d y \varepsilon(x-y) \psi_{i}(y), \psi_{j}\right)=\int_{\mathcal{I}} \int_{\mathcal{I}} d x d y \varepsilon(x-y) \psi_{i}(y) \psi_{j}(x),
$$

must now be calculated. The fact that $\varepsilon(x)=-\varepsilon(-x)$ implies

$$
B_{i j}=-B_{j i}, \quad B_{i i}=0,
$$

meaning that we only have to determine $n(2 n-1)$ matrix elements. Note that the elements are numbers depending on the parameters $N$ and $\beta$. In general the result for $S_{N}^{(\beta)}(x, y)$ in the scaling limit $N \rightarrow \infty$ has the highest interest. If an asymptotic relation for the orthonormal polynomials (and thus for $\psi_{i}$ ) is well known, it is therefore relevant to examine whether or not it is legal to put in the asymptotic relations when this limit is taken in (32). It is certainly preferable to interchange the integration and the limit $N \rightarrow \infty$ in (32), the justification of which is given by Lebesgue's Majorant Theorem. The general procedure is valid for all (even) $N$, though, and in the next steps we work with the general matrix $B$, with no reference to the actual calculation of the matrix elements.

(6) The matrices on the right hand sides of (15) and (16) are given by the $2 n \times 2 n$ matrices $J$ and $C$

$$
J_{i j} \equiv \delta_{i j}-\left.\left(\delta_{i j}\right)\right|_{i, j>n}, \quad 0 \leq i, j \leq 2 n,
$$

and

$$
C \equiv J+B A
$$


For instance for $n=1$ we have

$$
J=\left(\begin{array}{ll}
1 & 0 \\
0 & 0
\end{array}\right) .
$$

The matrix $A_{0}$ is defined by the $2 n \times n$ matrix produced by deleting the last $n$ columns in $A$ and the $n \times 2 n$ matrix $C_{0}$ is defined by the matrix produced by deleting the last $n$ rows in $C$. In addition we have the $n \times n$ matrix $C_{00}$ given by deleting the last $n$ rows and the last $n$ columns in $C$. We need only the $n$ last rows of the matrix

$$
A_{0} C_{00}^{-1} C_{0}
$$

and the $n$ first columns of

$$
A C(I-B A C)^{-1}
$$

(7) The functions $\varepsilon \psi_{i}(x)=\int_{\mathcal{I}} \varepsilon(x-y) \psi_{i}(y)$ are ingredients in the $n$ correction terms in (15) and in (16), as well as in the matrix elements $B_{i j}=\left(\varepsilon \psi_{i}, \psi_{j}\right)$. If allowed, it is highly preferable to put in the asymptotic relation for $\psi_{i}(x)$ in the scaling limit $N \rightarrow \infty$. We must solve the integrals explicitly if this is not allowed.

(8) The results of points (1), (4), (6) and (7) are collected to construct the right hand side of (15) and (16). For $\beta=4$ we must remember the replacement $N \rightarrow 2 N$ everywhere, giving the right hand side of (15).

\section{The kernels $S_{N}^{(\beta)}\left(x_{i}, x_{j}\right)$}

In this section we use the recipe of last section to derive the two kernels (15) and (16) in the the massless non- $\chi$ Gaussian ensemble, defined by the choice $m_{f}=0$ in the weight function (7).

For all $m_{f}=0$ we have the weight function

$$
\omega_{N_{f}}^{(\beta)}(x)=x^{N_{f}} e^{-c x^{2}}, \quad \beta=1,4,
$$

on the support $\mathcal{I}=]-\infty, \infty\left[\right.$, where $c=\frac{N \Sigma \beta}{4}$. The spectral density $\rho(x)$ is the 1-point correlation function and from (8) we have 任

$$
\rho(-x)=(-1)^{N N_{f}} \rho(x) .
$$

We will always take $N$ even making the spectral density an even function, also when $N_{f}$ is odd. For even $N_{f}$ the polynomials [20]

$$
\begin{aligned}
& H_{2 m}^{N_{f}}(x)=(-1)^{m}\left(\bar{h}_{m}^{N_{f}}\right)^{-\frac{1}{2}} c^{\frac{1+N_{f}}{4}} L_{m}^{\frac{N_{f}-1}{2}}\left(c x^{2}\right), \quad \bar{h}_{m}^{N_{f}}=\frac{\Gamma\left(\frac{N_{f}}{2}+m+\frac{1}{2}\right)}{m !}, \\
& H_{2 n+1}^{N_{f}}(x)=(-1)^{n}\left(\bar{h}_{n}^{N_{f}}\right)^{-\frac{1}{2}} c^{\frac{3+N_{f}}{4}} x L_{n}^{\frac{N_{f}+1}{2}}\left(c x^{2}\right), \quad \bar{h}_{n}^{N_{f}}=\frac{\Gamma\left(\frac{N_{f}}{2}+n+\frac{3}{2}\right)}{n !},
\end{aligned}
$$

are orthonormal with respect to $(39)$ on $\mathcal{I}=]-\infty, \infty\left[\right.$. Here the functions $L_{n}^{\alpha}(x)$ are the generalized Laguerre polynomials (which are orthonormal with respect to $\omega(x)=x^{\alpha} e^{-x}, \alpha>-1$, on $\left.\mathcal{I}=\right] 0, \infty[$ ), with the co efficient of $x^{n}$ given by

$$
k_{n}=(-1)^{n} / n !
$$

We call the polynomials (41) and (42) generalized Hermite polynomials. Notice that these polynomials satisfy

$$
H_{2 m}^{N_{f}}(-x)=H_{2 m}^{N_{f}}(x), \quad H_{2 n+1}^{N_{f}}(-x)=-H_{2 n+1}^{N_{f}}(x),
$$


and

$$
H_{N}^{N_{f}}(x)=\frac{1}{x} H_{N+1}^{N_{f}-2}(x)
$$

the latter property is easily derived from a basic relation between Laguerre polynomials. Letting $\bar{k}_{i}^{N_{f}}$ be the coefficient of the highest power in $H_{i}^{N_{f}}(x)$ (and remembering that $N$ is even) implies

$$
a_{N}=\frac{\bar{k}_{N-1}}{\bar{k}_{N}}=\frac{(-1)^{\frac{N-2}{2}} k_{\frac{N-2}{2}}}{(-1)^{\frac{N}{2}} k_{\frac{N}{2}}} \frac{\left(\bar{h}_{N-1}^{N_{f}}\right)^{-\frac{1}{2}}}{\left(\bar{h}_{N}^{N_{f}}\right)^{-\frac{1}{2}}} \frac{c^{\frac{N-2}{2}} c^{\frac{3+N_{f}}{4}}}{c^{\frac{N}{2}} c^{\frac{1+N_{f}}{4}}}=\left(\frac{N}{2}\right)^{\frac{1}{2}} c^{-\frac{1}{2}},
$$

where $k_{i}^{N_{f}}$ is the coefficient (43). The generalized Hermite polynomials are relevant for all three cases $\beta=1,2,4$ and even $N_{f}$. For $\beta=2,4$ and even $N_{f}$ we must use the polynomials (41) and (42) which are orthonormal with respect to (39), but for odd $N_{f}$ we cannot use the orthonormal polynomial method described earlier, due to the fact that there does not exist orthonormal polynomials with respect to an odd weight function defined on an even interval [21]. For $\beta=1$, however, we must use the orthonormal polynomials with respect to $\omega_{N_{f}}^{(\beta=1)}(x)^{2}=x^{2 N_{f}} e^{-2 c x^{2}}$, implying that we can choose $N_{f}$ both even and odd in this case. The relevant polynomials are given (41) and (42) with the replacements $N_{f} \rightarrow 2 N_{f}$ and $c \rightarrow 2 c$, which we will carry out at the end of derivations. Thus, in both cases $\beta=1,4$ the number $N_{f}\left(2 N_{f}\right)$ is even. According to the prescription of the case $\beta=4$ we will do the replacement $N \rightarrow 2 N$ at the end of the derivations.

Following the recipe described in last section we now construct the kernels $S_{N}^{(\beta)}\left(x_{i}, x_{j}\right), \beta=1,4$, defined by the weight function (39).

With the polynomials $H_{i}^{N_{f}}(x)$, given by (41) and (42), and $\omega_{N_{f}}^{(\beta)}(x)$, given by (39), we have

$$
\varphi_{i}(x)=H_{i}^{N_{f}}(x) \omega_{N_{f}}^{(\beta)}(x)^{\frac{1}{2}}
$$

$i=0,1, \ldots$, and $\mathcal{H}=\operatorname{span}\left\{\varphi_{0}, \varphi_{1}, \ldots, \varphi_{N-1}\right\}$. In each ensemble $\beta=1,4$ we now have the kernel $K_{N}(x, y)$, through (10).

Because $N_{f}$ is even in both ensembles we have $\omega_{N_{f}}^{(\beta)}(x)=e^{-c x^{2}+N_{f} \ln |x|}$. It follows then, that

$$
U(x, y)=\frac{V^{\prime}(x)-V^{\prime}(y)}{x-y}=\frac{N_{f}}{x y}+\frac{\left(c x^{2}\right)^{\prime}-\left(c y^{2}\right)^{\prime}}{x-y}=\frac{N_{f}}{x y}+2 c .
$$

The polynomial $H_{N-1}^{N_{f}}(x)$ does not have a constant term, see (42), and therefore the function (22) vanishes. We have

$$
\begin{gathered}
A_{N}(x)=a_{N} \int_{-\infty}^{\infty} d y H_{N}^{N_{f}}(y) H_{N-1}^{N_{f}}(y)\left(\frac{N_{f}}{x y}+2 c\right) y^{N_{f}} \exp \left(-c y^{2}\right) \\
=a_{N} \frac{N_{f}}{x} \int_{-\infty}^{\infty} d y H_{N}^{N_{f}}(y) p_{N-2}(y) y^{N_{f}} \exp \left(-c y^{2}\right)=0
\end{gathered}
$$

where $p_{N-2}(y)=H_{N-1}^{N_{f}}(y) / y$. The function (23) equals

$$
B_{N}(x)=a_{N} \int_{-\infty}^{\infty} d y\left(H_{N}^{N_{f}}(y)\right)^{2}\left(\frac{N_{f}}{x y}+2 c\right) y^{N_{f}} \exp \left(-c y^{2}\right)
$$


It follows from the orthonormality properties that the integral of the second term in (50) equals $2 c a_{N}$. The integral of the first term gives

$$
\begin{aligned}
& a_{N} \frac{N_{f}}{x} \int_{-\infty}^{\infty} d y\left(H_{N}^{N_{f}}(y)\right)^{2} y^{N_{f}-1} \exp \left(-c y^{2}\right) \\
& \quad=\left.a_{N} \frac{N_{f}}{x} \frac{1}{N_{f}} y^{N_{f}}\left(H_{N}^{N_{f}}(y)\right)^{2} \exp \left(-c y^{2}\right)\right|_{-\infty} ^{\infty}-a_{N} \frac{N_{f}}{x} \frac{1}{N_{f}} \int_{-\infty}^{\infty} d y y^{N_{f}}\left(H_{N}^{N_{f}}(y) \exp \left(-c y^{2}\right)\right)^{\prime} \\
& \quad=-a_{N} \frac{1}{x} \int_{-\infty}^{\infty} d y y^{N_{f}}\left[2 H_{N}^{N_{f}}(y) H_{N}^{\prime N_{f}}(y)-2 y c\left(H_{N}^{N_{f}}(y)\right)^{2}\right] \exp \left(-c y^{2}\right)=0,
\end{aligned}
$$

implying that

$$
B_{N}(x)=2 c a_{N}=\sqrt{2 N} c^{\frac{1}{2}}
$$

We then have the functions

$$
\begin{array}{r}
A(x)=-\frac{1}{2} V^{\prime}(x)=-\frac{1}{2}\left(2 c x-\frac{N_{f}}{x}\right)=-c x+\frac{N_{f}}{2 x}, \quad B_{N}(x)=2 c a_{N}=\sqrt{2 N} c^{\frac{1}{2}}, \\
C(x)=\frac{a_{N}}{a_{N-1}} B_{N-1}=2 c a_{N}=B_{N}(x)=\sqrt{2 N} c^{\frac{1}{2}} .
\end{array}
$$

Now we have the matrix in (26), making it possible to determine the matrix $A$ after the choice of a number $2 n$ of functions $\psi_{i}(x)$, see (30).

The function

$$
\frac{\frac{d}{d x} \omega_{N_{f}}^{(\beta)}(x)}{\omega_{N_{f}}^{(\beta)}(x)}=\frac{N_{f}}{x}-2 c x,
$$

has a pole at $x=0$ and at $x=\infty$, with orders $n_{0}=1$ and $n_{\infty}=1$ respectively. Thus we have $n=n_{\infty}+n_{0}=2$ meaning that we have to calculate two correction terms.

We must find $n=2$ linearly independent functions $\psi_{1}, \psi_{2} \in \mathcal{H}$ and 2 linearly independent $\psi_{3}, \psi_{4} \in \mathcal{H}^{\perp}$. These functions must be written as linear combinations of the functions (27) and (28), with only the case $k=0$, and each $\psi_{i}, i=1,2,3,4$, is therefore a linear combination of the four functions

$$
\varphi_{N-1}(x), \quad \varphi_{N}(x), \quad \frac{\varphi_{N-1}(x)}{x}, \quad \frac{\varphi_{N}(x)}{x} .
$$

The function $\frac{\varphi_{N-1}(x)}{x}$ is a polynomial of degree $(N-2)$ times $\omega_{N_{f}}^{(\beta)}(x)^{1 / 2}$ and thus it follows immediately that

$$
\psi_{1}(x) \equiv \varphi_{N-1}(x), \quad \psi_{2}(x) \equiv \frac{\varphi_{N-1}(x)}{x} \in \mathcal{H}
$$

In addition, with the help of orthogonality it is easily shown that

$$
\psi_{3}(x) \equiv \varphi_{N}(x), \quad \psi_{4}(x) \equiv \frac{\varphi_{N}(x)}{x} \in \mathcal{H}^{\perp} .
$$

The requirements are simply that $\psi_{3} \psi_{4} \notin \mathcal{H}$ and $\psi_{3}, \psi_{4} \perp \varphi_{0}$ and $\psi_{3}, \psi_{4} \perp \varphi_{N-1}$ (see (29)), which follows from orthogonality.

With the choices (56) and (57) we now determine the matrix $A$ through (26), (53) og (30). Inserting (53), (56) and (57), into (26) gives

$$
[D, K](x, y)=-a_{N}\left[c \varphi_{N}(x) \varphi_{N-1}(y)+c \varphi_{N-1}(x) \varphi_{N}(y)+\frac{N_{f}}{2 x y}\left(\varphi_{N}(x) \varphi_{N-1}(y)+\varphi_{N-1}(x) \varphi_{N}(y)\right)\right]
$$


A Comparison with (30) gives the matrix elements of the $4 \times 4$-matrix $A$. We get

$$
A=\left(\begin{array}{cccc}
0 & 0 & -a_{N} c & 0 \\
0 & 0 & 0 & -a_{N} \frac{N_{f}}{2} \\
-a_{N} c & 0 & 0 & 0 \\
0 & -a_{N} \frac{N_{f}}{2} & 0 & 0
\end{array}\right)=\left(\begin{array}{cccc}
0 & 0 & -\left(\frac{N}{2}\right)^{1 / 2} c^{\frac{1}{2}} & 0 \\
0 & 0 & 0 & -\left(\frac{N}{2}\right)^{1 / 2} c^{-\frac{1}{2}} \frac{N_{f}}{2} \\
-\left(\frac{N}{2}\right)^{1 / 2} c^{\frac{1}{2}} & 0 & 0 & 0 \\
0 & -\left(\frac{N}{2}\right)^{1 / 2} c^{-\frac{1}{2} \frac{N_{f}}{2}} & 0 & 0
\end{array}\right)
$$

which is symmetric and fulfills equation (31).

Because of (33) the $4 \times 4$-matrix $B$ is given by the elements

$$
\begin{gathered}
B_{12}=\left(\varepsilon \psi_{1}, \psi_{2}\right), \quad B_{13}=\left(\varepsilon \psi_{1}, \psi_{3}\right), \quad B_{14}=\left(\varepsilon \psi_{1}, \psi_{4}\right) \quad \text { and } \\
B_{23}=\left(\varepsilon \psi_{2}, \psi_{3}\right), \quad B_{14}=\left(\varepsilon \psi_{1}, \psi_{4}\right), \quad B_{34}=\left(\varepsilon \psi_{3}, \psi_{4}\right) .
\end{gathered}
$$

The generalized Hermite polynomials $H_{i}^{N_{f}}(x)$ are either even or odd functions and the same is true for the square root of the weight function $\omega_{N_{f}}^{(\beta)}(x)^{1 / 2}$ (because $N_{f}$ is even), and thus the functions $\psi_{i}(x)$, $i=1,2,3,4$, are even or odd. A general calculation of the inner product gives $(\varepsilon f, g)=0$, whenever $f$ and $g$ have same parity. From this it follows that

$$
B_{14}=\left(\varepsilon \psi_{1}, \psi_{4}\right)=B_{23}=\left(\varepsilon \psi_{2}, \psi_{3}\right)=0,
$$

which together with (33) results in

$$
B=\left(\begin{array}{cccc}
0 & \left(\varepsilon \psi_{1}, \psi_{2}\right) & \left(\varepsilon \psi_{1}, \psi_{3}\right) & 0 \\
-\left(\varepsilon \psi_{1}, \psi_{2}\right) & 0 & 0 & \left(\varepsilon \psi_{2}, \psi_{4}\right) \\
-\left(\varepsilon \psi_{1}, \psi_{3}\right) & 0 & 0 & \left(\varepsilon \psi_{3}, \psi_{4}\right) \\
0 & -\left(\varepsilon \psi_{2}, \psi_{4}\right) & -\left(\varepsilon \psi_{3}, \psi_{4}\right) & 0
\end{array}\right)
$$

We have

$B_{i j}=\left(\varepsilon \psi_{i}, \psi_{j}\right)=\int_{-\infty}^{\infty} d x \psi_{j}(x) \int_{-\infty}^{\infty} d y \varepsilon(x-y) \psi_{i}(y)=\int_{-\infty}^{\infty} d x \psi_{j}(x) \frac{1}{2}\left\{\left(\int_{-\infty}^{-x} d y+\int_{-x}^{x} d y\right)-\int_{x}^{a} d y\right\} \psi_{i}(y)$,

from which it follows that

$$
B_{i j}=2 \int_{0}^{\infty} d x \psi_{j}(x) \int_{0}^{x} d y \psi_{i}(y)
$$

whenever $\psi_{i}$ is an even function. Notice that in every element, $B_{i j} \neq 0$, one of the functions $\psi_{i}$ or $\psi_{j}$ is always even while the other is odd, which together with the feature $B_{i j}=-B_{j i}$ implies that the absolute value of all matrix elements $B_{i j}$ can be written on the form (65). Leaving these matrix elements unsolved for a while, we now focus on the structure of the kernels $S_{N}^{(\beta)}(x, y), \beta=1,4$.

Having the matrices $A, B$ and $(n=2)$

$$
J=\left(\begin{array}{llll}
1 & 0 & 0 & 0 \\
0 & 1 & 0 & 0 \\
0 & 0 & 0 & 0 \\
0 & 0 & 0 & 0
\end{array}\right), \quad C=J+B A, \quad A_{0}=\left(\begin{array}{cc}
0 & 0 \\
0 & 1 \\
-\left(\frac{N}{2}\right)^{1 / 2} c^{\frac{1}{2}} & 0 \\
0 & -\left(\frac{N}{2}\right)^{1 / 2} c^{-\frac{1}{2} \frac{\alpha}{2}}
\end{array}\right)
$$

and so on, the two kernels are given by

$$
S_{N}^{(4)}(x, y)=K_{N}(x, y)-\psi_{3}(x) \sum_{l=1}^{4}\left[A_{0} C_{00}^{-1} C_{0}\right]_{3 l} \varepsilon \psi_{l}(y)-\psi_{4}(x) \sum_{k=1}^{4}\left[A_{0} C_{00}^{-1} C_{0}\right]_{4 k} \varepsilon \psi_{k}(y)
$$




$$
S_{N}^{(1)}(x, y)=K_{N}(x, y)-\psi_{1}(x) \sum_{l=1}^{4}\left[A C(I-B A C)^{-1}\right]_{l 1} \varepsilon \psi_{l}(y)-\psi_{2}(x) \sum_{k=1}^{4}\left[A C(I-B A C)^{-1}\right]_{k 2} \varepsilon \psi_{k}(y),
$$

where

$$
\varepsilon \psi_{i}(x)=\frac{1}{2}\left\{\int_{-\infty}^{x} d y-\int_{x}^{\infty} d y\right\} \psi_{i}(y)=\frac{1}{2}\left\{\int_{-\infty}^{-x} d y+\int_{-x}^{x} d y-\int_{x}^{\infty} d y\right\} \psi_{i}(y),
$$

$i=1,2,3,4$. If $\psi_{i}$ is an even function we see that

$$
\varepsilon \psi_{i}(x)=\int_{0}^{x} d y \psi_{i}(y)
$$

while if $\psi_{i}$ is odd then

$$
\varepsilon \psi_{i}(x)=-\int_{x}^{\infty} d y \psi_{i}(y)
$$

Now we turn to the troublesome integrals in the kernels. The explicit expressions for the four functions $\psi_{i}(x)$ are given by $\varphi_{j}(x)=H_{j}^{(\beta)}(x) \omega_{N_{f}}^{(\beta)}(x)^{1 / 2},(56)$ and $(57)$ and are

$$
\begin{aligned}
& \psi_{1}(x)=(-1)^{\frac{N}{2}-1}\left[\frac{\left(\frac{N}{2}-1\right) !}{\Gamma\left(\frac{N}{2}+\frac{N_{f}+1}{2}\right)}\right]^{\frac{1}{2}} c^{\frac{3+N_{f}}{4}} L_{\frac{N}{2}-1}^{\frac{N_{f}+1}{2}}\left(c x^{2}\right) x^{\frac{N_{f}}{2}+1} e^{-c \frac{x^{2}}{2}}, \\
& \psi_{2}(x)=\frac{\psi_{1}(x)}{x}, \\
& \psi_{3}(x)=(-1)^{\frac{N}{2}}\left[\frac{\left(\frac{N}{2}\right) !}{\Gamma\left(\frac{N}{2}+\frac{N_{f}+1}{2}\right)}\right]^{\frac{1}{2}} c^{\frac{1+N_{f}}{4}} L_{\frac{N}{2}}^{\frac{N_{f}-1}{2}}\left(c x^{2}\right) x^{\frac{N_{f}}{2}} e^{-c \frac{x^{2}}{2}}, \\
& \psi_{4}(x)=\frac{\psi_{3}(x)}{x} .
\end{aligned}
$$

For relatively large $N$ it is clear that an actual calculation of the two kernels $S_{N}^{(\beta)}(x, y)$ becomes quite involved due to the presence of the integrals (65), (70) and (71). Actually, it is the microscopic limit of the kernel

$$
S_{N}^{(\beta)}(x, y)_{s} \equiv \lim _{N \rightarrow \infty} \frac{1}{N \Sigma} S_{N}^{(\beta)}\left(\frac{x}{N \Sigma}, \frac{y}{N \Sigma}\right), \quad \beta=1,4,
$$

giving all the microscopic correlations, which has physical interest. Therefore it is necessary to determine whether it is allowed to substitute an asymptotic relation for the polynomials in the integrals when the microscopic limit is taken. If the answer to this is positive, we can get rid of the $N$ dependence and derive a closed analytical expression for the microscopic kernel (73). With the help of Lebesgue's Majorant Theorem this question is pursued and answered in Appendix A. For (70), of course, the use of an asymptotic relation for $\psi_{i}$ is allowed when the microscopic limit is taken, because the integral is finite for all $N$. We must check that the second equality in the following equation

$$
\lim _{N \rightarrow \infty} N^{\lambda} B_{i j}=\lim _{N \rightarrow \infty} N^{\lambda} 2 \int_{0}^{\infty} d x \psi_{j}(x) \int_{0}^{x} d y \psi_{i}(y)=2 \int_{0}^{\infty} d x \lim _{N \rightarrow \infty} N^{\lambda} \psi_{j}(x) \int_{0}^{x} d y \psi_{i}(y),
$$

as well as the last equality in ( see (71))

$$
\lim _{N \rightarrow \infty} N^{\delta} \varepsilon \psi_{i}\left(\frac{x}{N}\right)=-\lim _{N \rightarrow \infty} N^{\delta} \int_{\frac{x}{N}}^{\infty} d y \psi_{i}(y)=-\lim _{N \rightarrow \infty} N^{\delta} \frac{1}{N} \int_{x}^{\infty} d u \psi_{i}\left(\frac{u}{N}\right)=-\int_{x}^{\infty} d u \lim _{N \rightarrow \infty} N^{\delta-1} \psi_{i}\left(\frac{u}{N}\right),
$$


are valid. Here we assume that the power of $N^{\lambda}$ and $N^{\delta}$ have the exact values needed to make the number $N^{\lambda} B_{i j}$ and the function $N^{\delta-1} \psi_{i}(x / N)$ convergent for $N \rightarrow \infty$. This is a consequence of the assumption that the microscopic kernel $(73)$ is finite.

Now let us examine (74) for $i=1$ and $j=2$. After two substitutions we have

$$
B_{12}=C_{N} \int_{0}^{\infty} d z L_{\frac{N}{2}-1}^{\frac{N_{f}+1}{2}}(2 z) z^{\frac{1}{2}\left(\frac{N_{f}}{2}-1\right)} e^{-z} \int_{0}^{z} d u L_{\frac{N^{2}}{2}-1}^{\frac{N_{f}+1}{2}}(2 u) u^{\frac{N_{f}}{4}} e^{-u}
$$

where all irrelevant factors have been collected in $C_{N}$. (We remember that (65) only holds when $\psi_{i}$ is an even function, meaning that $N_{f} / 2$ is odd in case of $i=1$ (see (72) ). But the fact $B_{i j}=-B_{j i}$ implies that all $B_{i j}$ can be represented on the form (65), when we keep track of the sign.) Using the result of Appendix A.2 we find that (74) does not hold for $B_{12}$. From (122) and point (2) at the end of A.2, this is a consequence of

$$
-\left(\frac{N_{f}+1}{4}+\frac{1}{4}-\frac{N_{f}}{4}\right)=-\frac{1}{2} \geq-1
$$

and

$$
-\left(\frac{N_{f}+1}{4}+\frac{1}{4}-\left(\frac{N_{f}}{4}-\frac{1}{2}\right)\right)=-1 \geq-1 .
$$

A similar treatment of the explicit expressions for the three other matrix elements $B_{13}, B_{24}$ and $B_{34}$ gives that the corresponding equation (74) does not hold for any of them. An undesirable consequence is that we have to solve all four integrals $B_{12}, B_{13}, B_{24}$ and $B_{34}$ before enlarging $N$ in the kernels (67) and $(68)$.

Turning to the question of the validity of the last equality in (75) we must use the result of Appendix A.1. Consider for instance $\psi_{1}$ and assume that this function is odd, that is $\left(\left(N_{f} / 2\right)+1\right)$ is odd. Then from $(72)$ and $(71)$ we have

$$
\begin{aligned}
\varepsilon \psi_{1}\left(\frac{x}{N}\right) & =-\frac{1}{N} \int_{x}^{\infty} d u \psi_{1}\left(\frac{u}{N}\right) \propto-\frac{1}{N} \int_{x}^{\infty} d u L_{\frac{N}{2}-1}^{\frac{N_{f}+1}{2}}\left(c \frac{u^{2}}{N^{2}}\right)\left(\frac{u}{N}\right)^{\frac{N_{f}}{2}+1} e^{-c \frac{u^{2}}{2 N^{2}}} \\
& \propto-\int_{x}^{\infty} d t L_{\frac{N}{2}-1}^{\frac{N_{f}+1}{2}}(2 t) t^{\frac{N_{f}}{4}} e^{-t}
\end{aligned}
$$

where all irrelevant factors have been skipped. From $(109)$ and $(120)$ it now follows that $(75)$ is not valid for $i=1$. This is caused by the fact

$$
\frac{N_{f}+1}{4}-\frac{N_{f}}{4}=\frac{1}{4} \leq \frac{3}{4}
$$

Considering the functions $\varepsilon \psi_{i}(x), i=2,3,4$, with $\psi_{i}(x)$ odd (which of course is not possible for all functions at the same time), an analogue argumentation gives the same conclusion. When the function $\psi_{i}(x), i=1,2,3,4$, is odd we therefore have to solve the integral $\varepsilon \psi_{i}(x)$ to make an exact calculation of the kernels (68) and (67) possible (within reasonable time) for large $N$.

We have learned that for finite $N$ a calculation of $B_{12}, B_{13}, B_{24}$ and $B_{34}$ and an analytical expression for the four functions $\varepsilon \psi_{i}(x), i=1,2,3,4$, are needed to make the results (68) and (67) usable. This requirement has been met in Appendix B.

In Appendix B.1 we derive an expression for the function

$$
\mathcal{E}_{[\bar{\alpha}, \bar{\beta}, n]}(x) \equiv \varepsilon L_{n}^{\bar{\alpha}}\left(x^{2}\right) x^{\bar{\beta}} e^{-\frac{x^{2}}{2}},
$$


for integers $n, \bar{\beta}$ and $\alpha>-1$. The functions $\varepsilon \psi_{i}(x), i=1,2,3,4$, belong to this class of functions and it is easily derived that (see (72))

$$
\begin{gathered}
\varepsilon \psi_{1}(x)=k_{1} c^{-\frac{1}{4}} \mathcal{E}_{\left[\frac{N_{f}+1}{2}, \frac{N_{f}}{2}+1, \frac{N}{2}-1\right]}\left(c^{\frac{1}{2}} x\right), \\
\varepsilon \psi_{2}(x)=k_{2} c^{\frac{1}{4}} \mathcal{E}_{\left[\frac{N_{f}+1}{2}, \frac{N_{f}}{2}, \frac{N}{2}-1\right]}\left(c^{\frac{1}{2}} x\right), \\
\left.\varepsilon \psi_{3}(x)=k_{3} c^{-\frac{1}{4}} \mathcal{E}_{\left[\frac{N_{f}-1}{2},\right.} \frac{N_{f}}{2}, \frac{N}{2}\right] \\
\left.\varepsilon \psi_{4}(x)=k_{4} c^{\frac{1}{4}} \mathcal{E}_{\left[\frac{N_{f}-1}{2},\right.}, \frac{N_{f}}{2}-1, \frac{N}{2}\right]
\end{gathered}
$$

with the coefficients

$$
\begin{aligned}
& k_{1}=(-1)^{\frac{N}{2}-1}\left[\frac{\left(\frac{N}{2}-1\right) !}{\Gamma\left(\frac{N}{2}+\frac{N_{f}+1}{2}\right)}\right]^{\frac{1}{2}}, \\
& k_{2}=k_{1}, \\
& k_{3}=(-1)^{\frac{N}{2}}\left[\frac{\left(\frac{N}{2}\right) !}{\Gamma\left(\frac{N}{2}+\frac{N_{f}+1}{2}\right)}\right]^{\frac{1}{2}}, \\
& k_{4}=k_{3} .
\end{aligned}
$$

For each $\psi_{i}$ the associated function $\mathcal{E}$ above is given by (144). From (72) it is clear that if $N_{f} / 2$ is even, then $\psi_{1}$ and $\psi_{4}$ are odd functions and $\psi_{2}$ and $\psi_{3}$ are even functions. For odd $N_{f} / 2$, of course, the situation is the other way around. Thus for each kernel (68) and (67) our derived expression will split into two parts, one for even $N_{f} / 2$ and one for odd $N_{f} / 2$.

In Appendix B.2 the result for the function $\mathcal{E}$ is used to calculate

$$
\mathcal{B}_{i j} \equiv\left(\varepsilon \phi_{i}, \phi_{j}\right)=\int_{-\infty}^{\infty} d x \phi_{j}(x) \int_{-\infty}^{\infty} d y \varepsilon(x-y) \phi_{i}(y)
$$

where

$$
\phi_{j}(x)=L_{m}^{\alpha}\left(x^{2}\right) x^{\beta} e^{-\frac{x^{2}}{2}} \quad \text { og } \quad \phi_{i}(x)=L_{n}^{\bar{\alpha}}\left(x^{2}\right) x^{\bar{\beta}} e^{-\frac{x^{2}}{2}},
$$

and $n, m, \beta, \bar{\beta}$ are integers, fulfilling certain conditions. We have always an odd $\bar{\beta}$, meaning that $\phi_{i}$ is odd. By keeping track of a sign we can always, with the help of $B_{i j}=-B_{j i}$ as described earlier, secure that the function in the inner integral of the double integral $B_{i j}=\left(\varepsilon \psi_{i}, \psi_{j}\right)$ is odd (or even). Thus the four relevant numbers $B_{i j}$ are contained in the derived expressions for (87) in Appendix B.2. Putting the functions (72) into (87), and remembering the mentioned possible change of sign when we secure that the odd function comes in the inner integral of $B_{i j}$, gives the following result for the four matrix elements of $B$ :

$$
\begin{gathered}
B_{12}=(-1)^{\frac{N_{f}}{2}} k_{1}^{2} \mathcal{B}_{12}, \\
B_{13}=(-1)^{\frac{N_{f}}{2}} c^{-\frac{1}{2}} k_{1} k_{3} \mathcal{B}_{13}, \\
B_{24}=(-1)^{\frac{N_{f}}{2}+1} c^{\frac{1}{2}} k_{2} k_{4} \mathcal{B}_{24}, \\
B_{34}=(-1)^{\frac{N_{f}}{2}+1}\left(k_{3}\right)^{2} \mathcal{B}_{34},
\end{gathered}
$$

where the numbers $\mathcal{B}_{i j}$ are found with the help of table 2 and 3 . 


\begin{tabular}{||c|c|c||}
\hline \hline even $\frac{N_{f}}{2}$ & given by eq. & with parameters \\
\hline \hline $\mathcal{B}_{12}$ & $(159)$, without the $(+)$-term & $\alpha, \bar{\alpha}=\frac{N_{f}+1}{2}, \beta=\frac{N_{f}}{2}, \bar{\beta}=\frac{N_{f}}{2}+1, n=m=\frac{N}{2}-1$ \\
\hline $\mathcal{B}_{13}$ & $(\overline{159})$, without the $(+)$-term & $\alpha=\frac{N_{f}-1}{2}, \bar{\alpha}=\frac{N_{f}+1}{2}, \beta=\frac{N_{f}}{2}, \bar{\beta}=\frac{N_{f}}{2}+1, m=\frac{N}{2}, n=\frac{N}{2}-1$ \\
\hline $\mathcal{B}_{24}$ & $(\overline{164})$ & $\alpha=\frac{N_{f}+1}{2}, \bar{\alpha}=\frac{N_{f}-1}{2}, \beta=\frac{N_{f}}{2}, \bar{\beta}=\frac{N_{f}}{2}-1, m=\frac{N}{2}-1, n=\frac{N}{2}$ \\
\hline $\mathcal{B}_{34}$ & $(159)$, without the $(+)$-term & $\alpha=\bar{\alpha}=\frac{N_{f}-1}{2}, \beta=\frac{N_{f}}{2}-1, \bar{\beta}=\frac{N_{f}}{2}, m=n=\frac{N}{2}$ \\
\hline
\end{tabular}

Table 2: A recipe giving $\mathcal{B}_{i j}$ for even $N_{f} / 2$.

\begin{tabular}{||c|c|c||}
\hline \hline odd $\frac{N_{f}}{2}$ & given by eq. & with parameters \\
\hline \hline $\mathcal{B}_{12}$ & $(159)$, without the $(+)$-term & $\alpha, \bar{\alpha}=\frac{N_{f}+1}{2}, \beta=\frac{N_{f}}{2}+1, \bar{\beta}=\frac{N_{f}}{2}, n=m=\frac{N}{2}-1$ \\
\hline $\mathcal{B}_{13}$ & $(159)$, including the $(+)$-term & $\alpha=\frac{N_{f}+1}{2}, \bar{\alpha}=\frac{N_{f}-1}{2}, \beta=\frac{N_{f}}{2}+1, \bar{\beta}=\frac{N_{f}}{2}, m=\frac{N}{2}-1, n=\frac{N}{2}$ \\
\hline $\mathcal{B}_{24}$ & $(159)$, without the $(+)$-term & $\alpha=\frac{N_{f}-1}{2}, \bar{\alpha}=\frac{N_{f}+1}{2}, \beta=\frac{N_{f}}{2}-1, \bar{\beta}=\frac{N_{f}}{2}, m=\frac{N}{2}, n=\frac{N}{2}-1$ \\
\hline $\mathcal{B}_{34}$ & $(159)$, without the (+)-term & $\alpha=\bar{\alpha}=\frac{N_{f}-1}{2}, \beta=\frac{N_{f}}{2}, \bar{\beta}=\frac{N_{f}}{2}-1, m=n=\frac{N}{2}$ \\
\hline
\end{tabular}

Table 3: A recipe giving $\mathcal{B}_{i j}$ for odd $N_{f} / 2$.

Having calculated the four matrix elements of $B$ and the four functions $\varepsilon \psi_{i}$ we can now construct the two kernels (68) and (67) of the non- $\chi$ Gaussian ensemble. The proceeding points summarize the construction of the kernels :

(1) Through equation (10), the kernel $K_{N}(x, y)$ is given by (47) with the associated comments.

(2) Assume that $\left(\frac{N_{f}}{2}\right)$ is even (odd). Then it follows from (72), that $\psi_{1}$ and $\psi_{4}$ are odd (even), and $\psi_{2}$ and $\psi_{3}$ are even (odd). The functions $\varepsilon \psi_{1}, \varepsilon \psi_{2}, \varepsilon \psi_{3}$ and $\varepsilon \psi_{4}$ are given by (82), (83), (84) and (85), respectively, with $\mathcal{E}$ given by (144) both for even and odd $\psi_{i}$.

(3) The matrix elements $B_{12}, B_{13}, B_{24}$ and $B_{34}$ depend on whether $N_{f} / 2$ is even or odd. These are given by (89), (90), (91) and (92), with $\mathcal{B}_{i j}$ given by tables 2 and 3 . Matrix $B$ is given by (63).

(4) The matrix

$$
C=J+B A
$$

is given by (59), (63) and (66). We need the matrices

$$
\begin{aligned}
A_{0} C_{00}^{-1} C_{0} & \text { for } & \beta=4, & \text { and } \\
A C(I-B A C)^{-1} & \text { for } & \beta & =1 .
\end{aligned}
$$

(5) Before putting all pieces together in the construction of (68) and (67) we remember the replacements

$$
\begin{array}{lll}
\beta=1 & : \quad c \rightarrow 2 c \\
\beta=4 & : \quad & N \rightarrow 2 N,
\end{array} \quad \text { and } \quad N_{f} \rightarrow 2 N_{f},
$$

in all parts above and in the functions (72). In the two ensembles $\beta=1,4$ we have $c=\frac{N \Sigma^{2} \beta}{4}$.

The five points above leads to two formulas for each $S_{N}^{(\beta)}(x, y), \beta=1,4$ : one for even $N_{f}$ and one for odd $N_{f}$ for $\beta=1$, and one for even $N_{f} / 2$ and one for odd $N_{f} / 2$ for $\beta=4$. 


\section{$5 \quad$ The microscopic spectral density}

In the microscopic limit the spectral correlations between the eigenvalues of the Dirac operator in the two Yang-Mills theories mentioned in the introduction can be derived from the corresponding symplectic or orthogonal non- $\chi$ Gaussian ensembles. In this section we present our results for our numerical expressions for the microscopic spectral density.

From (14) it follows that the 1-point correlation function, that is the spectral density, is given by

$$
\rho^{(\beta)}(x) \equiv R_{N}^{(\beta)}(x)=S_{N}^{(\beta)}(x, x), \quad \beta=1,4 .
$$

The machinery from the last section gives both $S_{N}^{(\beta)}(x, y), \beta=1,4$, and we therefore have in principle all correlation functions and especially the microscopic spectral density in the cases $\beta=1$ and $\beta=4$ of the non- $\chi$ Gaussian ensemble. The microscopic spectral density is defined by

$$
\rho_{s}^{(\beta)}(x) \equiv \lim _{N \rightarrow \infty} \frac{1}{N} \rho^{(\beta)}\left(\frac{x}{N}\right), \quad \beta=1,4,
$$

and this is of physical interest. We have not yet extracted the $N$ dependence of our results for the $B_{i j}$ (derived in appendix B.2) and for the functions $\varepsilon \psi_{i}$ (derived in appendix B.1) in the microscopic limit and we therefore have not an exact analytical result for $\rho_{s}^{(\beta)}(x), \beta=1,4$. However, we can easily present plots of the scaled spectral density

$$
\rho_{N}^{(\beta)}(x) \equiv \frac{1}{N} \rho^{(\beta)}\left(\frac{x}{N}\right)=\frac{1}{N} S_{N}^{(\beta)}\left(\frac{x}{N}, \frac{x}{N}\right), \quad \beta=1,4,
$$

which, of course, converges towards $\rho_{s}^{(\beta)}(x)$ for large $N$.

Due to the presence of the Vandermonde determinant in the probability distribution one expects that the spectrum becomes more rigid when the parameter $\beta$ is increased (see (8)). Thus we naturally expect the scaled spectral density $\rho_{N}^{(1)}(x)$ in the ensemble non- $\chi$ GOE to be flat compared to $\rho_{N}^{(2)}$ in non- $\chi$ GUE, and that $\rho_{N}^{(4)}$ in non- $\chi$ GSE is the most oscillating function of the three of them. These features are of course valid for all values of $N$.

In both ensembles $\beta=1,4$ the kernel $S_{N}^{(\beta)}(x, y)$ is equal to a kernel $K_{(2) N}(x, y)$ from the unitary ensemble $\beta=2$ (remembering the modifications associated to each value of $\beta=1,4$ ), plus $n$ corrections, in general (see (15) and (16)). Thus in the two non- $\chi$ ensembles we have $\rho_{N}^{(\beta)}(x)=\rho_{N}^{(2)}(x)+\rho_{N}^{(\beta)}$ corr. $(x)$, $\beta=1,4$, where $\rho_{N}^{(2)}(x)=\left(N^{-1}\right) K_{(2) N}\left(N^{-1} x, N^{-1} x\right)$ and $\rho_{N \text { corr }}^{(\beta)}(x)$ constitute the 2 correction terms. When having calculated the matrix $B$ and the four functions $\varepsilon \psi_{i}(x)$ both scaled corrections $\rho_{N}^{(\beta)}$ corr. $(x), \beta=1,4$, are given by the two last terms on the right hand side of (67) and (68). The contribution from the scaled spectral density $\rho_{N}^{(2)}(x)$ is an oscillating function in both ensembles $\beta=1,4$, and the flat spectrum of $\rho_{N}^{(1)}(x)$ in the ensemble non- $\chi$ GOE is therefore expected to be a result of the fact that $\rho_{N}^{(1)}$ corr. $(x)$ precisely cuts off the peaks of $\rho_{N}^{(2)}(x)$, and at the same time the term $\rho_{N}^{(4)}$ corr. $(x)$ must cause a highly oscillating $\rho_{N}^{(4)}(x)$. When plotting $\rho_{N}^{(\beta)}(x)$ together with the associated $\rho_{N}^{(2)}(x)$ we indeed observe these highly non-trivial features. The results are illustrated in figure 11 and 2.

In the limit $N \rightarrow \infty$ we have $\rho_{N}^{(\beta)}(x) \rightarrow \rho_{s}^{(\beta)}(x), \beta=1,4$. This convergence, however, is especially fast for $x$ close to zero. This is illustrated in figure 3, where we have plotted $\rho_{N}^{(\beta)}(x), \beta=1,4$, for different values of $N$. The spectral density is an even function (see (40)) and for finite $N$ we have the so-called half circles (with a hole in the vicinity of $x=0$, due to the term $x^{N_{f}}$ in the weight function), which 



Figure 1: In non- $\chi$ GOE we have $\rho_{N}^{(1)}(x)=\rho_{N}^{(2)}(x)+\rho_{N}^{(1)}$ corr. $(x)$, where $\rho_{N}^{(2)}(x)$ is from the associated unitary ensemble (given by orthonormal polynomials with respect to $\left.\omega_{N_{f}}^{(1)}(x)^{2}\right)$ and $\rho_{N}^{(1)}$ corr. $(x)$ is the scaled correction derived in last section. In left column the oscillating term $\rho_{40}^{(2)}(x)$ and the entire $\rho_{40}^{(1)}(x)$ are plotted together for $N=40$ and the two values $N_{f}=2$ (above) and $N_{f}=4$ (under). The corresponding correction terms $\rho_{40}^{(1)}$ corr. $(x)$ are plotted in right column. We observe that the flat spectrum of non- $\chi$ GOE is exactly a result of $\rho_{40}^{(1)}$ corr. $(x)$, cutting of the peaks of $\rho_{40}^{(2)}(x)$. In addition the curve of $\rho_{40}^{(1)}(x)$ moves away from zero when $N_{f}$ is enlarged, due to the presence of the determinant function in the probability distribution (see (3)). 

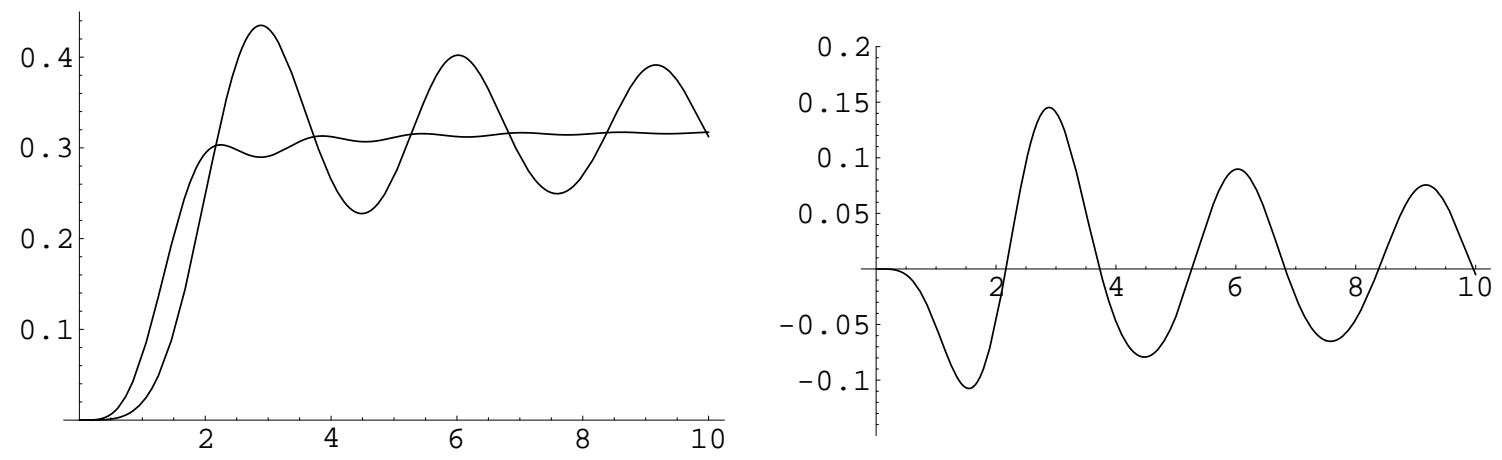

Figure 2: In non- $\chi$ GSE we have $\rho_{N}^{(4)}(x)=\rho_{N}^{(2)}(x)+\rho_{N}^{(4)}$ corr. $(x)$, where $\rho_{N}^{(2)}(x)$ is from the associated unitary ensemble (given by orthonormal polynomials with respect to $\omega_{N_{f}}^{(4)}(x)$ and the replacement $N \rightarrow 2 N)$ and $\rho_{N}^{(4)}$ corr. $(x)$ is the scaled correction derived in last section. On the left the term $\rho_{40}^{(2)}(x)$ and the entire $\rho_{40}^{(1)}(x)$ are plotted together for $N=40$ and the value $N_{f}=4$. The corresponding correction term $\rho_{40}^{(4)}$ corr. $(x)$ is plotted on the right. We observe that $\rho_{40}^{(4)}$ corr. $(x)$ gives a highly oscillating $\rho_{40}^{(4)}(x)$ (a rigid spectrum).

is clearly seen in figure 3. Because of the the Gaussian distribution the microscopic spectral density fulfills $\rho_{s}(x) \rightarrow \pi^{-1}$ for $x \rightarrow \infty$ and thus the curves $\rho_{N}^{(\beta)}(x), \beta=1,4$, are of order $\sim \pi^{-1}$ before the half circles start approaching zero (see also figure 1 and 2).
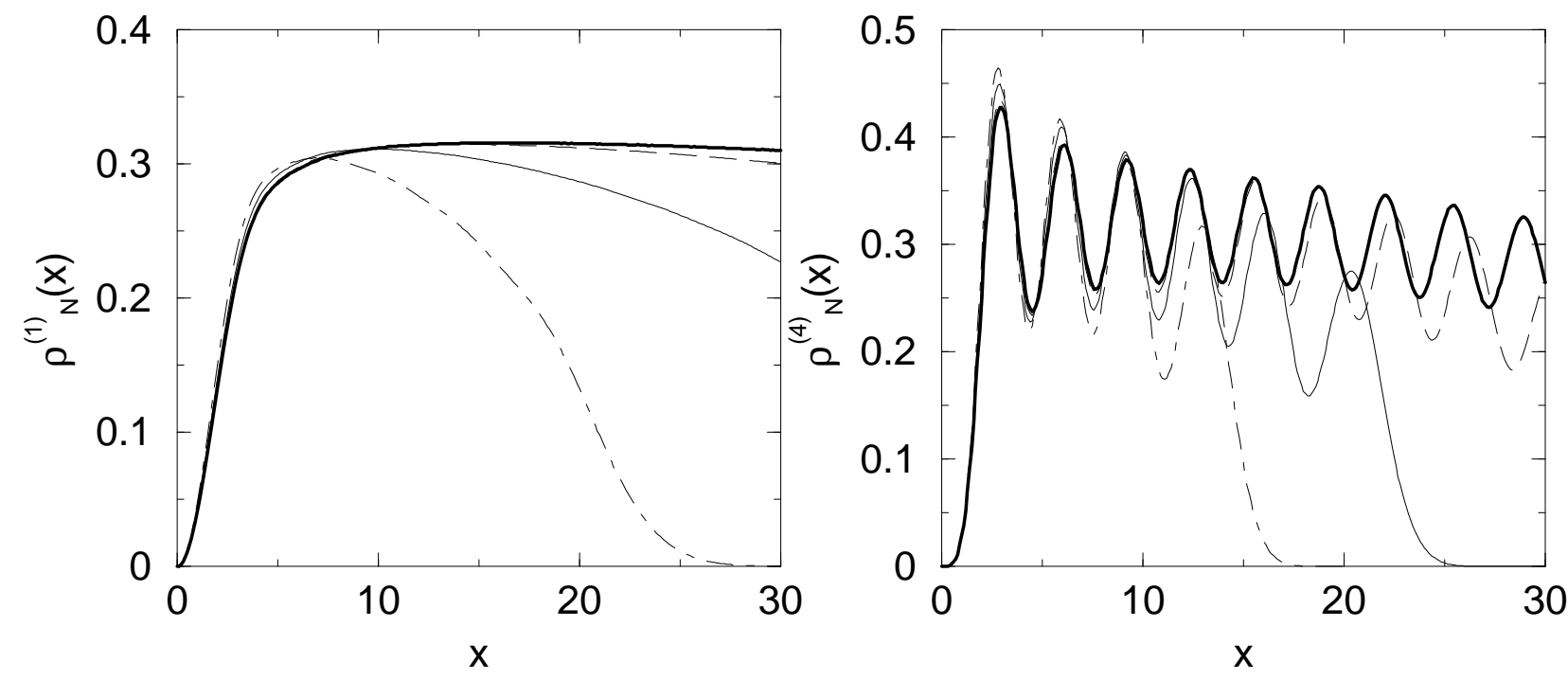

Figure 3: The scaled spectral density $\rho_{N}^{(\beta)}(x)$, for increasing $N$, on the left for $\beta=1$ and on the right for $\beta=4$. The convergens in the vicinity of $x=0$ is clearly observed and we have $\rho_{N}^{(\beta)}(x) \approx \rho_{s}^{(\beta)}(x)$ near $z=0$ as a consequence. In the limit $N \rightarrow \infty$ we have $\rho_{N}^{(\beta)}(x) \rightarrow \rho_{s}^{(\beta)}(x)$ and in the limit $x \rightarrow \infty$ we have $\rho_{s}^{(\beta)}(x) \rightarrow \pi^{-1}$. 


\subsection{The first spectral sum rule}

The spectral sum rules derived in field theory are all given by the microscopic spectral density $\rho_{s}$ of the Dirac operator [7, 27] and the result for $\rho_{s}$ derived from RMT can therefore be verified through the knowledge of a spectral sum rule. The first sum involves the sum over the eigenvalues of the Dirac operator in second inverse power and in the limit $N \rightarrow \infty$ we have trivially

$$
\left\langle\sum_{\lambda_{k} \neq 0} \frac{1}{\left(N \Sigma \lambda_{k}\right)^{2}}\right\rangle=\int_{-\infty}^{\infty} d z \frac{\rho_{s}(z)}{z^{2}},
$$

where the sum is over all $\lambda_{k} \neq 0$ (both positive and negative) and the average is taken over all gauge fields. For both field theories corresponding to the ensembles $\beta=1,4$ in $(2+1)$ dimensions (see table 11) the same spectral sum rule has been derived in [10, 11] and it reads

$$
\left\langle\sum_{\lambda_{k} \neq 0} \frac{1}{\left(N \Sigma \lambda_{k}\right)^{2}}\right\rangle=\frac{4}{N_{f}} .
$$

According to this, the first spectral sum rule involving the eigenvalues of the Dirac operator in two completely different field theories in $(2+1)$ dimensions should be identical.

With the help of our numerical expressions for $\rho_{s}^{(\beta)}(x)$ for the microscopic spectral density $\rho_{s}^{(\beta)}(x)$ in the two ensembles $\beta=1,4$ we thus have a consistency check on our results through (99) and (100). From figure 2 and 3 it is easily seen that the effect of the finite $N$ is very small for a relatively large $N$. As seen our results does not agree with the spectral sum rule (100). In the ensemble $\beta=4$ it

\begin{tabular}{||c|c|c||c||}
\hline \hline$N_{f}$ & $\int_{-\infty}^{\infty} d z \frac{\rho_{40}^{(1)}(z)}{z^{2}}$ & $\int_{-\infty}^{\infty} d z \frac{\rho_{40}^{(4)}(z)}{z^{2}}$ & $\frac{4}{N_{f}}$ \\
\hline \hline 2 & 0.402 & 0.946 & 2 \\
\hline 4 & 0.148 & 0.436 & 1 \\
\hline 6 & 0.092 & 0.297 & 0.667 \\
\hline 8 & 0.067 & 0.227 & 0.500 \\
\hline 12 & 0.044 & 0.155 & 0.333 \\
\hline \hline
\end{tabular}

Table 4: The calculated first spectral sum rule, i.e the right hand side of (99), in the two ensembles $\beta=1$ and $\beta=4$ together with the spectral sum rule (100).

seems the spectral sum rule approaches $2 / N_{f}$ for large $N_{f}$.

Next we turn to the most elementary check of our results, which in fact is an actual generation of matrices.

\section{The Monte Carlo simulation}

We have performed a Monte Carlo simulation in order to numerically verify the derived scaled spectral densities $\rho_{N}^{(\beta)}(x), \beta=1,4$ The matrices in the generated ensemble are distributed according to (3), with all $m_{f}=0$. The simulations are done for various values of the matrix size, $N$, and different values of $N_{f}$ in the two cases $\beta=1,4$.

We diagonalize matrices from an ensemble made by a simple Metropolis algorithm. The eigenvalues, 

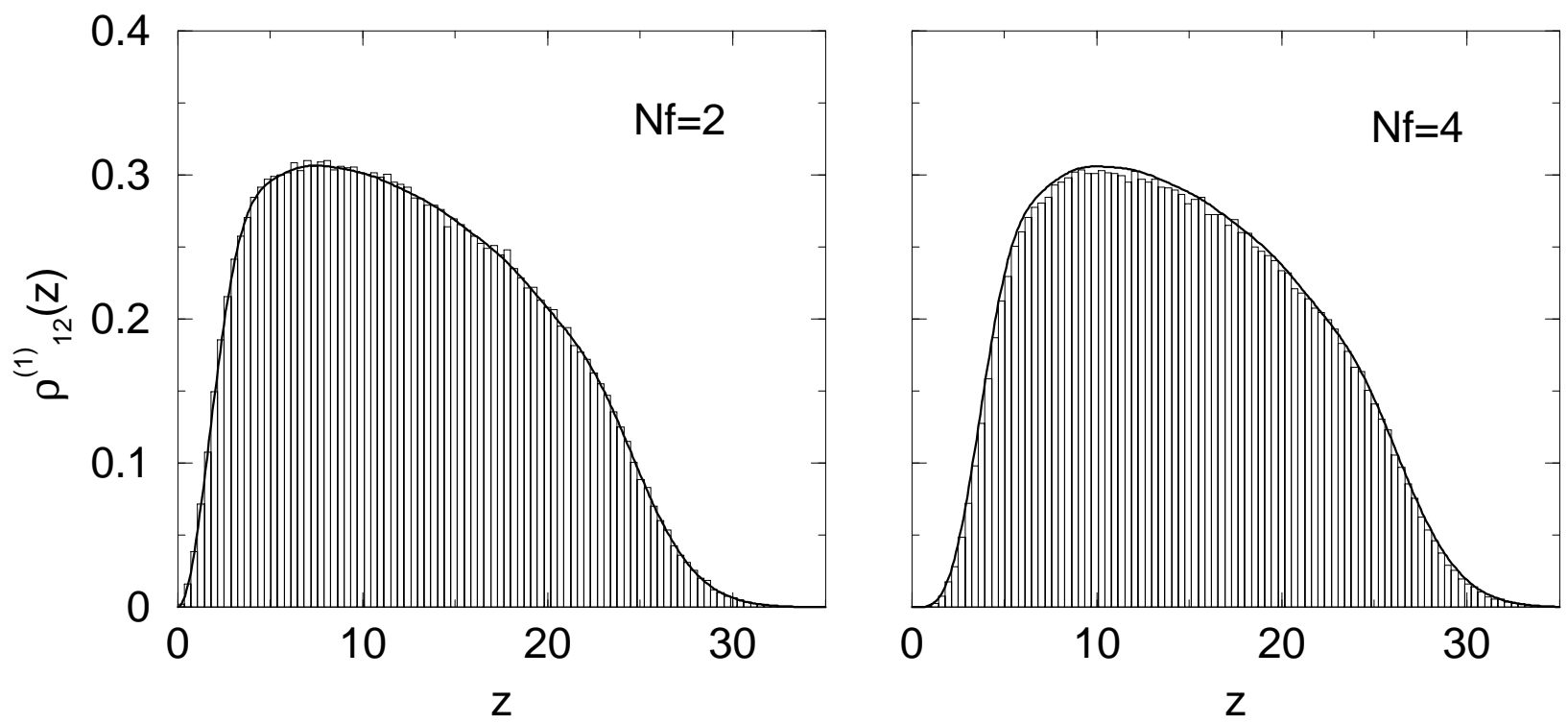

Figure 4: Scaled spectral density of the non- $\chi$ GOE ensemble, compared with the Monte Carlo data, $N=12, N_{f}=2$ and 4 .

$x$, are then collected to form a histogram, $h(x)$, which is rescaled

$$
\begin{aligned}
& x \rightarrow x N \equiv z, \\
& h \rightarrow \frac{h}{N}
\end{aligned}
$$

to obtain $\frac{1}{N} h\left(\frac{z}{N}\right)$. The rescaled histogram is then compared to the plot of the analytically obtained scaled spectral density. Note that we compare the analytic plots for finite $N$ with the corresponding simulation data. We thus expect the plots to match for the whole spectrum. For large $N$ the scaled spectral density close to $z=0$ has the form of the microscopic spectral density.

We handle the two cases $\beta=1,4$ individually as follows.

\section{$6.1 \beta=1$}

The Metropolis step consist in changing the entries in the matrix and accepting these changes according to the action

$$
S(M)=\frac{N}{4} \operatorname{Tr} M^{2}-N_{f} \ln (\operatorname{det} M) .
$$

The changes is done in a way that explicitly maintain the symmetry of the matrix $\left(M=M^{T}\right)$.

In this way we obtained an ensemble of 100,000 effectively uncorrelated matrices for $N=12$ and 10,000 matrices for $N=40$, both for $N_{f}=2$ and 4 . The resulting histograms together with their analytic equivalents can be seen in figure 4 and 5 . We observe excellent agreement in all parts of the spectrum, for all values of the parameters $N$ and $N_{f}$.

\section{$6.2 \beta=4$}

In this case the Metropolis steps are accepted/rejected according to

$$
S(M)=N \operatorname{Tr} M^{2}-N_{f} \ln (\operatorname{det} M)
$$



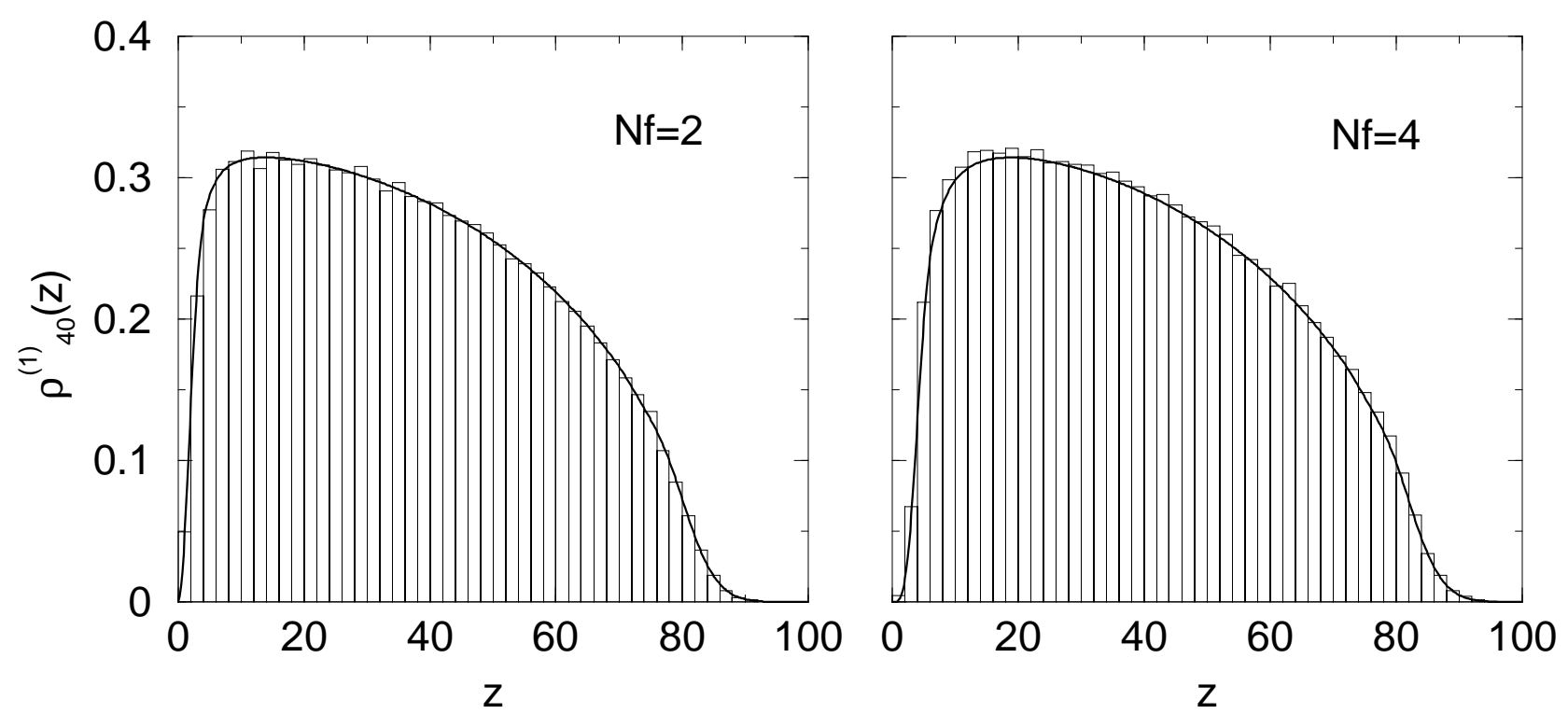

Figure 5: Scaled spectral density of the non- $\chi$ GOE ensemble, compared with the Monte Carlo data, $N=40, N_{f}=2$ and 4 .

A matrix from the symplectic ensemble can be represented by a $2 N \times 2 N$ matrix, $M$. This matrix can be written as a sum of direct-product matrices

$$
M=M_{0} e_{0}+M_{1} e_{1}+M_{2} e_{2}+M_{3} e_{3}
$$

where

$$
\begin{aligned}
& e_{0}=\left(\begin{array}{ll}
1 & 0 \\
0 & 1
\end{array}\right), \quad e_{1}=\left(\begin{array}{cc}
0 & i \\
i & 0
\end{array}\right) \\
& e_{2}=\left(\begin{array}{cc}
0 & -1 \\
1 & 0
\end{array}\right), \quad e_{3}=\left(\begin{array}{cc}
i & 0 \\
0 & -i
\end{array}\right)
\end{aligned}
$$

and the coefficients $M_{1}, M_{2}, M_{3}$ are real antisymmetric $N \times N$ matrices and $M_{0}$ is a real symmetric $N \times N$ matrix. In this way the matrix $M$ has $2 N$ eigenvalues which are doubly degenerate.

The Metropolis update works on the four real matrices, $M_{i}$, and $M$ is then constructed from these and diagonalized. In this the way the symmetry and structure of the matrices is kept at all times. For the histogram only the $N$ different eigenvalues is used.

When working in this $2 N \times 2 N$ representation the action, (103), has to be changed. The trace gets twice as large and the determinant is squared in this representation, so an overall factor of $1 / 2$ should be included:

$$
S(M)=\frac{N}{2} \operatorname{Tr} M^{2}-\frac{N_{f}}{2} \ln (\operatorname{det} M)
$$

These simulations are about twice as slow, so the maximum matrix size is half as big. We have done runs for $N=8$ and 20, with $N_{f}=2$ and 4 , with the same statistics as in the $\beta=1$ case. The plots are shown in figure 6 and 7 . Here the agreement is even more striking. Zooming in on the first few eigenvalues in the $N=20$ case we see absolutely perfect agreement indeed, see figure 8 . 

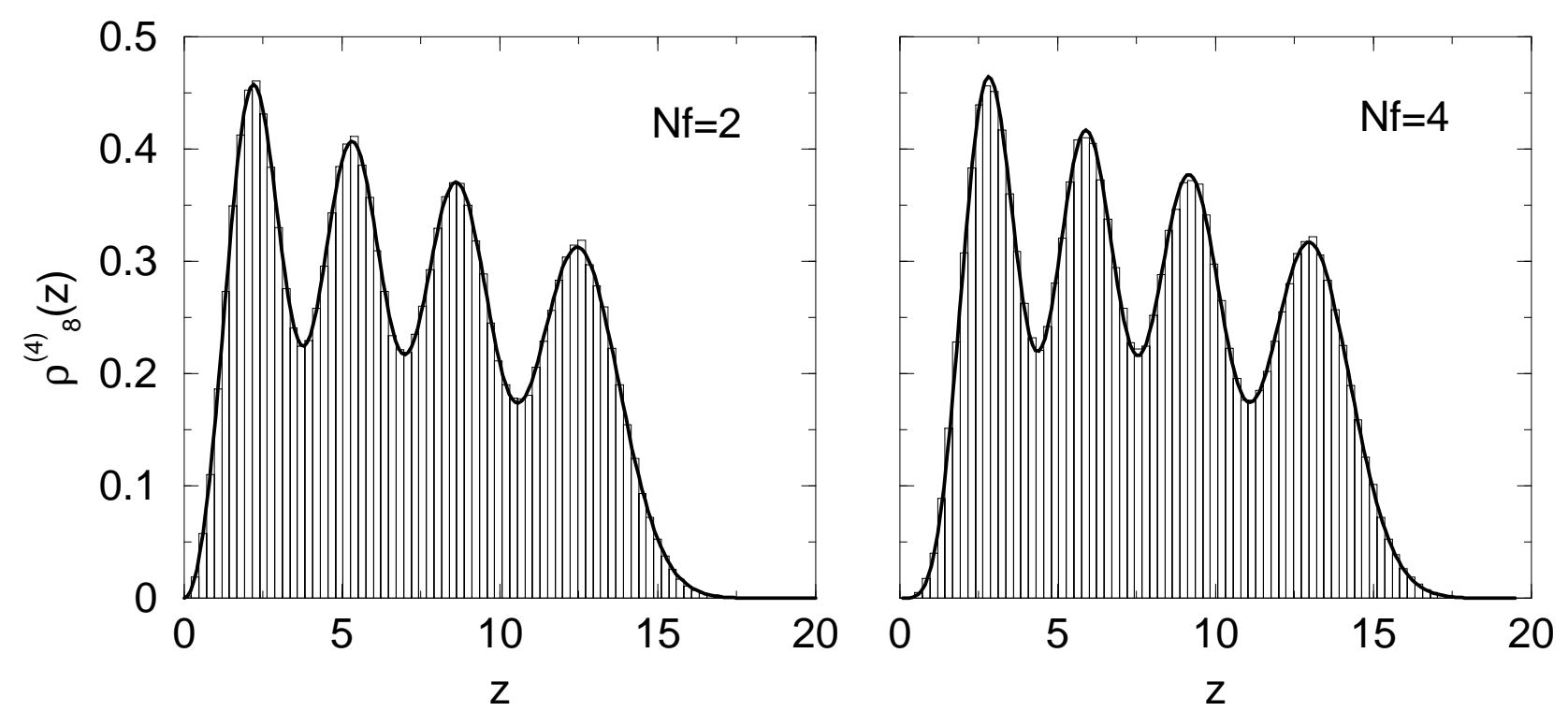

Figure 6: Scaled spectral density of the non- $\chi$ GSE ensemble, compared with the Monte Carlo data, $N=8, N_{f}=2,4$.
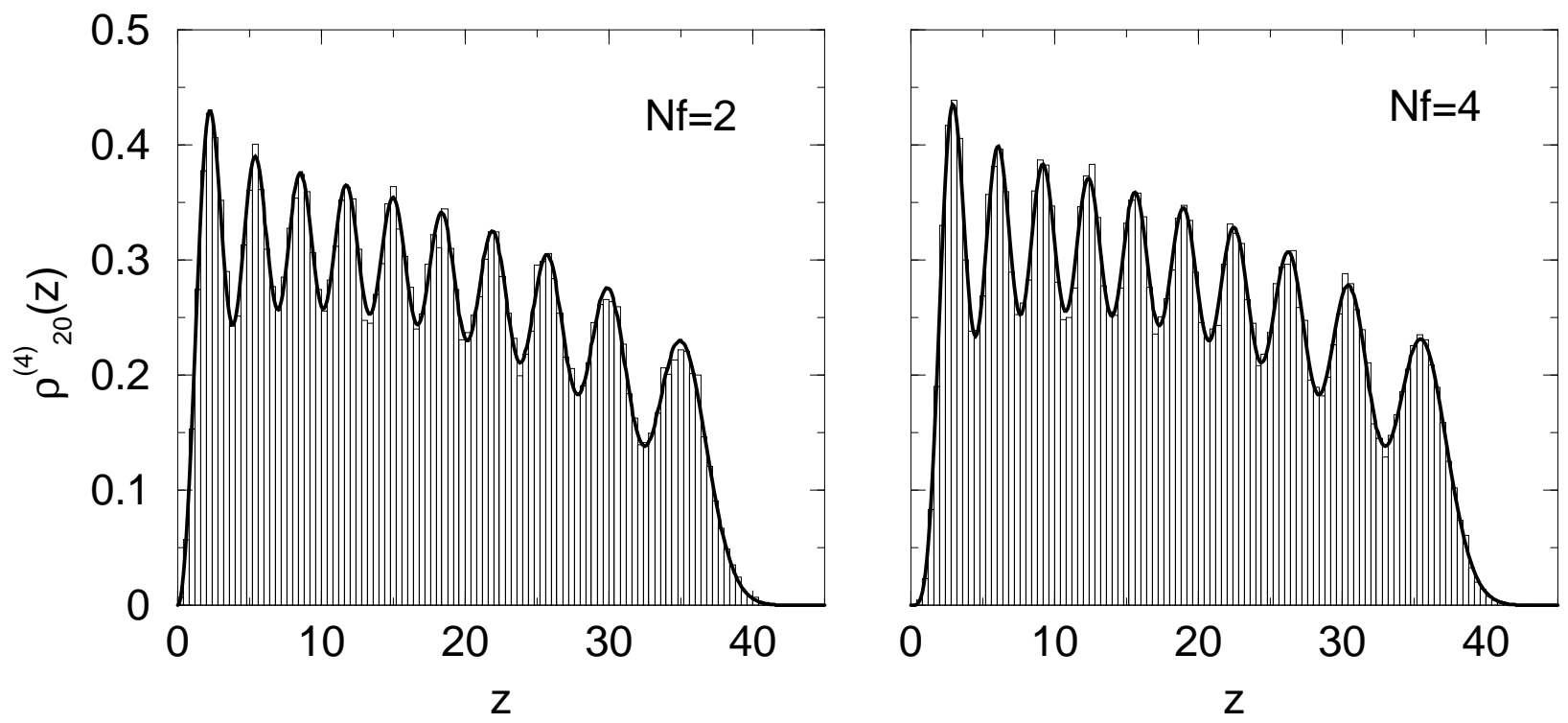

Figure 7: Scaled spectral density of the non- $\chi$ GSE ensemble, compared with the Monte Carlo data, $N=20, N_{f}=2,4$. 

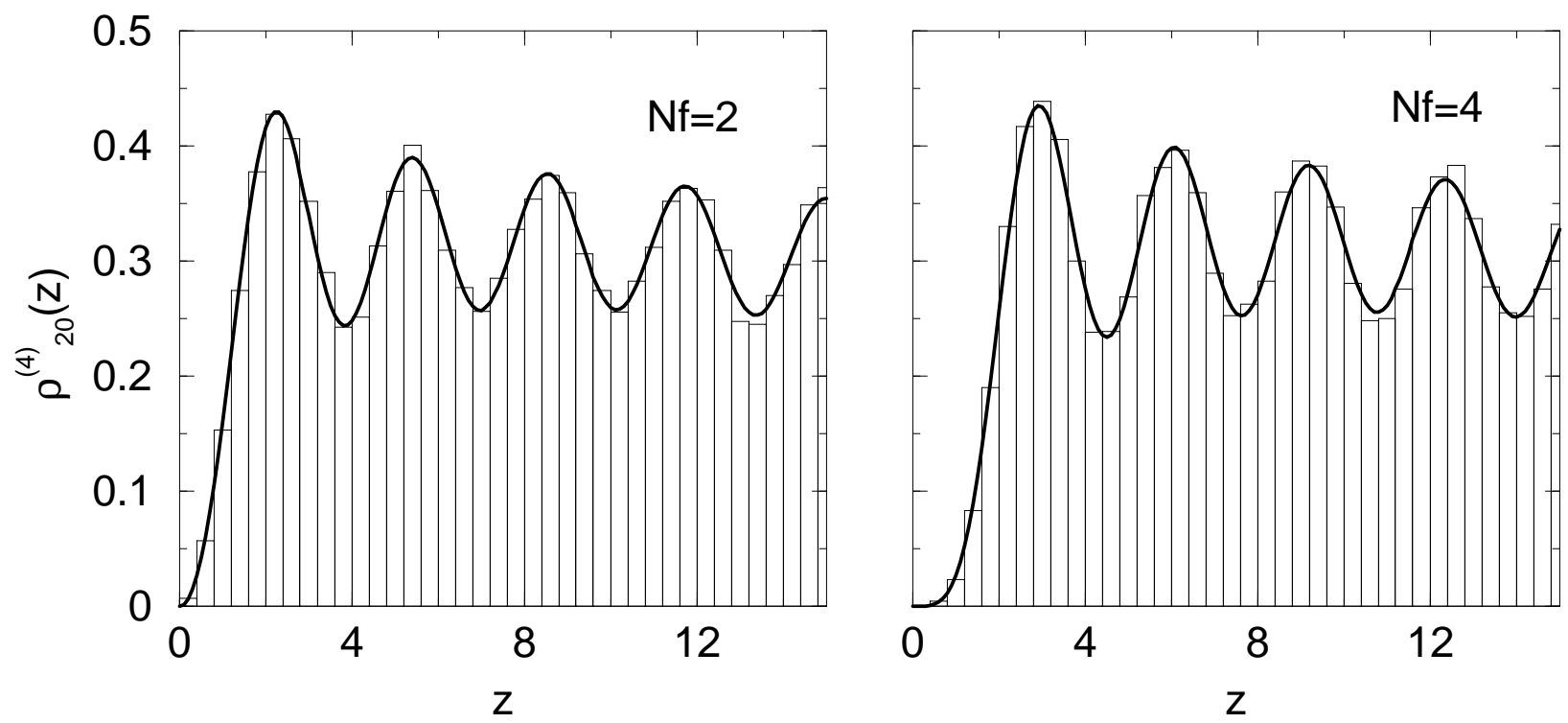

Figure 8: Same plot as figure 7 but zoomed in on small values of $z$.

\section{Conclusions}

The purpose of this study has been to derive the kernels $S_{N}^{(\beta)}(x, y), \beta=1,4$, from which all spectral correlation functions of the non- $\chi$ Gaussian orthogonal (non- $\chi$ GOE) and symplectic (non- $\chi$ GSE) ensembles can be determined. In the microscopic limit the two ensembles describes the microscopic spectral correlations of the low energy Dirac operator in two Yang-Mills theory in $(2+1)$ dimensions. Thus the universality class non- $\chi$ GOE carries information about the spectral correlations in a $S U(2)$ gauge theory with fundamental fermions, while the universality class non- $\chi$ GSE does the same for the $S U\left(N_{c}\right)$ gauge theory, with $N_{c}$ arbitrary, and fermions in the adjoint representation.

With the help of Widom's new method we have derived the kernels $S_{N}^{(\beta)}(x, y)$, in non- $\chi \operatorname{GOE}(\beta=1)$ and in non- $\chi$ GSE $(\beta=4)$ for massless fermions. For non- $\chi$ GOE our result are valid for all integers $N_{f}$ and for non- $\chi$ GSE we have a result for even $N_{f}$. We plotted the scaled spectral density $\rho_{N}^{(\beta)}(x)=N^{-1} S_{N}^{(\beta)}\left(N^{-1} x, N^{-1} x\right)$, for different values of the parameters $N$ and $N_{f}$. The plots of $\rho_{N}^{(\beta)}(x)$ possesses the expected features, a flat spectrum for $\beta=1$ and a highly oscillation spectrum for $\beta=4$, and indeed we see perfect agreement with our computer simulated spectra. For large $N$ the scaled spectral density $\rho_{N}^{(\beta)}(x)$ coincides with the microscopic spectral density $\rho_{s}(x)$ in the vicinity of $x=0$. Our calculated spectral sum rules from $\rho_{N}^{(\beta)}(x)$ of course match the computer generated ones perfectly. We have not found agreement with the sum rules of [10, 11].

Recently the microscopic spectral densities of the massive non- $\chi$ GOE and non- $\chi$ GSE have been derived by a different method [29]. In the massless limit these result seem to match ours for large $N$.

Acknowledgements : Poul Henrik Damgaard is thanked for many fruitful discussions. The work of C. Hilmoine is partially supported by a Rosenfeld Fellowship. 


\section{A Interchange of integration and the microscopic limit}

In this Appendix we look at the large $n$-behavior of two types of general integrals involving generalized Laguerre polynomials. Specifically we examine when interchange of integration and the microscopic limit is allowed. To this end Lebesgue's Majorant Theorem is used. The first section is relevant for the calculation of $\varepsilon \psi_{i}$ in the limit $N \rightarrow \infty$, see (75). The result of section 2 is relevant for the calculation of the elements of the matrix $B$ in the limit $N \rightarrow \infty$, see (74).

$$
\text { A.1 } \lim _{n \rightarrow \infty} \int_{0}^{\infty} d y n^{(1+a)-\alpha} L_{n}^{\alpha}(2 y) y^{a} \exp (-y)
$$

We define

$$
\mathcal{I}_{n}^{\alpha, a} \equiv \int_{0}^{\infty} d y n^{(1+a)-\alpha} L_{n}^{\alpha}(2 y) y^{a} \exp (-y)
$$

where $n$ is a positive integer, $a>-1$ and $\alpha$ are real numbers. We wish to calculate

$$
\lim _{n \rightarrow \infty} \mathcal{I}_{n}^{\alpha, a}=\lim _{n \rightarrow \infty} \int_{0}^{\infty} d y n^{(1+a)-\alpha} L_{n}^{\alpha}(2 y) y^{a} \exp (-y)
$$

and therefore examine when the interchange of the limit and the integration is allowed. In order to do this we employ Lebesgue's Majorant Theorem. At first we convince ourselfs that the sequence of functions in the integrand is convergent. The integral in (109) is rewritten by the substitution $u=(2 n y)^{1 / 2}$, and we get

$$
\lim _{n \rightarrow \infty} \mathcal{I}_{n}^{\alpha, a}=\lim _{n \rightarrow \infty} \int_{0}^{\infty} d u n^{(1+a)-\alpha} L_{n}^{\alpha}\left(\frac{u^{2}}{n}\right) u^{2 a+1} n^{-a-1} 2^{-a} \exp \left(-\frac{u^{2}}{2 n}\right) .
$$

The integrand in (110)

$$
f_{n}(u) \equiv n^{(1+a)-\alpha} L_{n}^{\alpha}\left(\frac{u^{2}}{n}\right) u^{2 a+1} n^{-a-1} 2^{-a} \exp \left(-\frac{u^{2}}{2 n}\right),
$$

is convergent for $n \rightarrow \infty$ : Inserting the well-known asymptotic formula 28

$$
\lim _{n \rightarrow \infty} \frac{1}{n^{\alpha}} L_{n}^{\alpha}\left(\frac{x^{2}}{n}\right)=x^{-\alpha} J_{\alpha}(2 x),
$$

we get

$$
\lim _{n \rightarrow \infty} f_{n}(u)=\lim _{n \rightarrow \infty} n^{(1+a)-\alpha} L_{n}^{\alpha}\left(\frac{u^{2}}{n}\right) u^{2 a+1} n^{-a-1} 2^{-a} \exp \left(-\frac{u^{2}}{2 n}\right)=2^{-a} u^{2 a+1-\alpha} J_{\alpha}(2 u) .
$$

Thus, the integrand in (109) is convergent. We must now look for an integrable majorant $\mathcal{M}(y)$, fulfilling for all $n$

$$
\left|f_{n}(y)\right|=\left|n^{(1+a)-\alpha} L_{n}^{\alpha}(2 y) y^{a} \exp (-y)\right|<\mathcal{M}(y) .
$$

If we can find such a function $\mathcal{M}(y)$, the Lebesgue Theorem states that interchange of the limit and integration is allowed in (109). In this case we can insert (113) in (109).

We start by splitting up the integral into integrals, in the first integrating over the interval $] 0, \epsilon[$ for some finite $\epsilon$ and in the second over ] $\epsilon, \infty$ [. Since the first integral is finite for every $n$ we focus only on the second integral. By choosing a sufficiently large $n^{\prime}$ we can use an asymptotic expression for the integrand. We then check if the absolute value of this expression has an integrable majorant for all $n>n^{\prime}$, see (114). Using the asymptotic property (valid for $x>0$ ) [25]

$$
L_{n}^{\alpha}(x)=\frac{1}{\pi} e^{\frac{x}{2}} x^{-\left(\frac{2 \alpha-1}{4}\right)} n^{\left(\frac{2 \alpha-1}{4}\right)} \cos \left[2(n x)^{\frac{1}{2}}-\frac{\alpha \pi}{2}-\frac{\pi}{4}\right]+\mathcal{O}\left(n^{\frac{2 \alpha-3}{4}}\right) .
$$


for $L_{n}^{\alpha}(2 y)$ in (109), we get

$$
\begin{aligned}
& \lim _{n \rightarrow \infty} \mathcal{I}_{n}^{\alpha, a} \\
& \approx \lim _{n \rightarrow \infty} \int_{\epsilon}^{\infty} d y n^{(1+a-\alpha)} \\
& \times\left\{\pi^{-1 / 2} \exp (y) y^{-\left(\frac{2 \alpha+1}{4}\right)} 2^{-\left(\frac{2 \alpha+1}{4}\right)} n^{\left(\frac{2 \alpha-1}{4}\right)} \cos \left[2(2 n y)^{\frac{1}{2}}-\frac{\alpha \pi}{2}-\frac{\pi}{4}\right]\right\} y^{a} \exp (-y) \\
&= \pi^{-1 / 2} 2^{-\left(\frac{2 \alpha+1}{4}\right)} \lim _{n \rightarrow \infty} n^{\left(1+a-\frac{\alpha}{2}-\frac{1}{4}\right)} \int_{\epsilon}^{\infty} d y y^{-\frac{2 \alpha+1}{4}+a} \cos \left[2(2 n y)^{\frac{1}{2}}-\frac{\alpha \pi}{2}-\frac{\pi}{4}\right],
\end{aligned}
$$

for $n>n^{\prime}$.

We know that the sequence of functions in (116) is convergent, and the function

$$
\mathcal{M}(y)=c \pi^{-1 / 2} 2^{-\left(\frac{2 \alpha+1}{4}\right)} y^{-\frac{2 \alpha+1}{4}+a}, \quad c>1,
$$

is then a majorant to the absolute value of the integrand in (109) for $n>n^{\prime}$ and some $c>1$, which take account of the term $\mathcal{O}$ in (115). Since we need $\int_{\epsilon}^{\infty} d y \mathcal{M}(y)<\infty$ we must have $-(\alpha / 2+1 / 4)+a<-1$. So we conclude, if

$$
\alpha / 2-a>3 / 4,
$$

then (117) is a integrable majorant and we can then interchange the order of the limit and the integral in (109). Asumming this in (113), gives us

$$
\lim _{n \rightarrow \infty} \mathcal{I}_{n}^{\alpha, a}=2^{-a} \int_{0}^{\infty} d u u^{2 a+1-\alpha} J_{\alpha}(2 u)
$$

for $\alpha / 2-a>3 / 4$.

On the contrary, if $\alpha / 2-a \leq 3 / 4$, then the integrand in (109) has no majorant, $\mathcal{M}(y)$, which fulfills $\int_{0}^{\infty} d y \mathcal{M}(y)<\infty$. This follows from the fact that the integral from $\epsilon$ to infinite of the absolut value of the integrand in (116) is not finite. Substituting $z=2 n y$ in (116) gives an integrand on the form $z^{-\delta} \cos \left(2 z^{1 / 2}+k\right)$, where $\delta=-(\alpha / 2+1 / 4)+a$ (the $n$ dependence vanishes of course). The smallest majorant to $\left|z^{-\delta} \cos \left(2 z^{1 / 2}+k\right)\right|$, is $C\left|z^{-\delta} \cos \left(2 z^{1 / 2}+k\right)\right|$, for some $C>1$. This majorant is not integrable when $\delta=-(\alpha / 2+1 / 4)+a \geq-1$, and thus we have no integrable majorant. When the absolut value of the asymptotic function does not integrate to a finite number, then of course no majorant exist for which the integral is finite (see (114)).

We conclude, when

$$
\alpha / 2-a \leq 3 / 4,
$$

interchange of integration and the limit in (109) is not legal. In this case we first have to solve the integral in (109) and subsequently derive an expression in the limit $n \rightarrow \infty$.

$$
\text { A.2 } \lim _{n \rightarrow \infty} \int_{0}^{\infty} d t n^{\lambda} L_{n}^{\alpha}(2 t) t^{\beta} \exp (-t) \int_{0}^{t} d u L_{n}^{\bar{\alpha}}(2 u) u^{\bar{\beta}} \exp (-u)
$$

We will now look at the limit $n \rightarrow \infty$ of integrals of the following type

$$
\mathcal{I}_{n}^{\alpha, \beta, \bar{\alpha}, \bar{\beta}} \equiv n^{\lambda} \int_{0}^{\infty} d t L_{n}^{\alpha}(2 t) t^{\beta} \exp (-t) \int_{0}^{t} d u L_{n}^{\bar{\alpha}}(2 u) u^{\bar{\beta}} \exp (-u)
$$

where $\alpha, \bar{\alpha}, \beta, \bar{\beta}>-1$ and $\lambda$ is real and $n$ is a positive integer. The matrix $B$ in section 5 is given by integrals of this type, and we are interested in an expression for $B$ in the limit $n \rightarrow \infty$. It is assumed that the integrand is convergent for $n \rightarrow \infty$, meaning that $\lambda$ has a certain value, the size of which is 
irrelevant for the question addressed in this Appendix.

Analogous to the previous section we investigate whether the limit and the integration can be interchanged in the expression

$$
\lim _{n \rightarrow \infty} \mathcal{I}_{n}^{\alpha, \beta, \bar{\alpha}, \bar{\beta}}=\lim _{n \rightarrow \infty} \int_{0}^{\infty} d t n^{\lambda} L_{n}^{\alpha}(2 t) t^{\beta} \exp (-t) \int_{0}^{t} d u L_{n}^{\bar{\alpha}}(2 u) u^{\bar{\beta}} \exp (-u) .
$$

By assumtion the sequence

$$
f_{n}(t) \equiv n^{\lambda} L_{n}^{\alpha}(2 t) t^{\beta} \exp (-t) \int_{0}^{t} d u L_{n}^{\bar{\alpha}}(2 u) u^{\bar{\beta}} \exp (-u),
$$

is convergent ( compare with (111) og (113) ). We must determine whether or not the absolut value of the integrandt in (122), that is $\left|f_{n}\right|$, has an integrabel majorant $\mathcal{M}(t)$. Like in Appendix B.1 we persuit this question by choosing a large $n$ and put in the asymptotic relation (115) for both Laguerre polynomials :

$$
\begin{aligned}
\lim _{n \rightarrow \infty} \mathcal{I}_{n}^{\alpha, \beta, \alpha, \bar{\beta}} \approx \pi^{-1} 2^{-\frac{\alpha+\bar{\alpha}+1}{2}} \lim _{n \rightarrow \infty} n^{\lambda} \times \\
\quad \int_{\epsilon}^{\infty} d t\left|n^{\frac{\alpha}{2}-\frac{1}{4}} t^{-\left(\frac{\alpha}{2}+\frac{1}{4}-\beta\right)} \cos \left[2(2 n t)^{\frac{1}{2}}-\frac{\alpha \pi}{2}-\frac{\pi}{4}\right] \int_{\epsilon}^{t} d u n^{\frac{\bar{\alpha}}{2}-\frac{1}{4}} u^{-\left(\frac{\bar{\alpha}}{2}+\frac{1}{4}-\bar{\beta}\right)} \cos \left[2(2 n u)^{\frac{1}{2}}-\frac{\bar{\alpha} \pi}{2}-\frac{\pi}{4}\right]\right| .
\end{aligned}
$$

Like in Appendix B.1 we skip all contributions involving irrelevant integrals over $] 0, \epsilon[$, because in these cases the exact integrals are finite. The sequence in (122), that is $f_{n}$ above, is convergent by assumtion. Thus for all $n$ the absolut value of the integrand in (124) is always smaller than the function

$$
c t^{-\left(\frac{\alpha}{2}+\frac{1}{4}-\beta\right)} \int_{\epsilon}^{t} d u u^{-\left(\frac{\bar{\alpha}}{2}+\frac{1}{4}-\bar{\beta}\right)},
$$

where $c>1$. Letting $\delta=-\left(\frac{\alpha}{2}+\frac{1}{4}-\beta\right)$ and $\rho=-\left(\frac{\bar{\alpha}}{2}+\frac{1}{4}-\bar{\beta}\right)$ we have trivially

$$
c \int_{\epsilon}^{\infty} d t t^{\delta} \int_{\epsilon}^{t} d u u^{\rho}=\frac{c}{\rho+1} \int_{\epsilon}^{\infty} d t\left(t^{\rho+\delta+1}-t^{\delta} \epsilon^{\rho+1}\right) .
$$

We see immediately that for

$$
\rho+\delta+1=-\frac{\alpha+\bar{\alpha}-1}{2}+\beta+\bar{\beta}<-1 \quad \text { and } \quad \delta=-\left(\frac{\alpha}{2}+\frac{1}{4}-\beta\right)<-1
$$

the integral in (126) is finite. For large $n$ we therefore have that the absolute value of the integrand in (123) always is smaller than the function (125), and if (127) is valid the function (125) is an integrable majorant.

On the contrary we see from (124) in case of $\delta=-\left(\frac{\alpha}{2}+\frac{1}{4}-\beta\right) \geq-1$ and $\rho=-\left(\frac{\bar{\alpha}}{2}+\frac{1}{4}-\bar{\beta}\right) \geq-1$, then the integral over $t$ is divergent. It follows that the absolut value of the integrand in (122) does not have an integrable majorant, since the asymptotic expression does not even have one.

The other cases of values of $\rho$ and $\delta$ are not relevant for us and we ignore them.

Summarizing the discussion we have

(1) If (127) is valid, then the absolut value of the integrand in (122) has an integrable majorant (125). Thus interchange of the limit and integration in (122) is legal:

$$
\lim _{n \rightarrow \infty} \mathcal{I}_{n}^{\alpha, \beta, \alpha, \bar{\beta}}=\int_{0}^{\infty} d t \lim _{n \rightarrow \infty} n^{\lambda} L_{n}^{\alpha}(2 t) t^{\beta} \exp (-t) \int_{0}^{t} d u L_{n}^{\bar{\alpha}}(2 u) u^{\bar{\beta}} \exp (-u) .
$$


(2) If $-\left(\frac{\alpha}{2}+\frac{1}{4}-\beta\right) \geq-1$ and $-\left(\frac{\bar{\alpha}}{2}+\frac{1}{4}-\bar{\beta}\right) \geq-1$, then the absolut value of the integrand in (122) has no majorant $\mathcal{M}(t)$ fulfilling $\int_{0}^{\infty} d t \mathcal{M}(t)<\infty$. Therefore interchange of the limit and the integration in (122) is not allowed. In this case we must solve the integral before the limit is taken.

\section{B Two integrals solved}

In this Appendix we find expressions for two different types of integrals. We wish to derive an expression for the function

$$
\mathcal{E}_{[\bar{\alpha}, \bar{\beta}, n]}(x) \equiv \varepsilon L_{n}^{\bar{\alpha}}\left(x^{2}\right) x^{\bar{\beta}} \exp \left(-\frac{x^{2}}{2}\right)=\int_{-\infty}^{\infty} d y \varepsilon(x-y) L_{n}^{\bar{\alpha}}\left(y^{2}\right) y^{\bar{\beta}} \exp \left(-\frac{y^{2}}{2}\right),
$$

where $\bar{\alpha}>-1$ is a real number and $n, \bar{\beta}$ are integers.

For integers $m$ and $n$ we calculate the following numbers

$$
\begin{aligned}
\mathcal{B}_{i j} & \equiv\left(\varepsilon L_{n}^{\bar{\alpha}}\left(x^{2}\right) x^{\bar{\beta}} \exp \left(-\frac{x^{2}}{2}\right), L_{m}^{\alpha}\left(x^{2}\right) x^{\beta} e^{-\frac{x^{2}}{2}}\right)=\int_{-\infty}^{\infty} d x L_{m}^{\alpha}\left(x^{2}\right) x^{\beta} e^{-\frac{x^{2}}{2}} \varepsilon L_{n}^{\bar{\alpha}}\left(x^{2}\right) x^{\bar{\beta}} e^{-\frac{y^{2}}{2}} \\
& =\int_{-\infty}^{\infty} d x L_{m}^{\alpha}\left(x^{2}\right) x^{\beta} e^{-\frac{x^{2}}{2}} \int_{-\infty}^{\infty} d y \varepsilon(x-y) L_{n}^{\bar{\alpha}}\left(y^{2}\right) y^{\bar{\beta}} e^{-\frac{y^{2}}{2}}
\end{aligned}
$$

The index $i$ refers to $m, \alpha$, and $\beta$, while $j$ refers to $n, \bar{\alpha}$ and $\bar{\beta} . m, n, \beta$ and $\bar{\beta}$ are integers and $\alpha$ and $\bar{\alpha}$ are real numbers greater than -1 .

The function (129) is a part of the inner product in $\mathcal{B}_{i j}$ and first we therefore derive an expression for (129).

B.1 $\varepsilon L_{n}^{\bar{\alpha}}\left(x^{2}\right) x^{\bar{\beta}} \exp \left(-\frac{x^{2}}{2}\right)$

We start by defining

$$
E(\lambda, x) \equiv \varepsilon x^{\lambda}\left(x e^{-\frac{x^{2}}{2}}\right),
$$

where $\lambda \geq-1$ is an integer. We have the following recursion formula

$$
\begin{aligned}
E(\lambda, x) & =\int_{-\infty}^{\infty} d y \varepsilon(x-y) y^{\lambda}\left(y e^{-\frac{y^{2}}{2}}\right) \\
& =\frac{1}{2}\left[\int_{-\infty}^{x} d y-\int_{x}^{\infty} d y\right] y^{\lambda}\left(y e^{-\frac{y^{2}}{2}}\right) \\
& =-\left[\frac{1}{2} e^{-\frac{y^{2}}{2}} y^{\lambda}\right]_{-\infty}^{x}+\left[\frac{1}{2} e^{-\frac{y^{2}}{2}} y^{\lambda}\right]_{x}^{\infty}+\int_{-\infty}^{\infty} d y \varepsilon(x-y) \lambda y^{\lambda-1}\left(e^{-\frac{y^{2}}{2}}\right) \\
& =-e^{-\frac{x^{2}}{2}} x^{\lambda}+\lambda \int_{-\infty}^{\infty} d y \varepsilon(x-y) y^{\lambda-2}\left(y e^{-\frac{y^{2}}{2}}\right) \\
& =-e^{-\frac{x^{2}}{2}} x^{\lambda}+\lambda E(\lambda-2, x),
\end{aligned}
$$

for $\lambda \geq 1$. For $\lambda=-1$ we have

$$
\begin{aligned}
E(-1, x) & =\int_{-\infty}^{\infty} d y \varepsilon(x-y) e^{-\frac{y^{2}}{2}} \\
& =\frac{1}{2}\left\{\int_{-\infty}^{-x} d y+\int_{-x}^{x} d y-\int_{x}^{\infty}\right\} e^{-\frac{y^{2}}{2}} \\
& =\int_{0}^{x} d y e^{-\frac{x^{2}}{2}}=\sqrt{\frac{\pi}{2}} \operatorname{erf}\left(\frac{x}{\sqrt{2}}\right) .
\end{aligned}
$$


And for $\lambda=0$ we get

$$
E(0, x)=\int_{-\infty}^{\infty} d y \varepsilon(x-y) y e^{-\frac{y^{2}}{2}}=-e^{-\frac{x^{2}}{2}}
$$

For $\lambda$ even we immediately get from (132) and (134)

$$
E(\lambda, x)=-e^{-\frac{x^{2}}{2}}\left(x^{\lambda}+\lambda x^{\lambda-2}+\ldots+\lambda(\lambda-2) \cdots 4 \cdot 2\right) .
$$

In the case of odd $\lambda,(132)$ and (133) give us :

$$
E(\lambda, x)=-e^{-\frac{x^{2}}{2}}\left(x^{\lambda}+\lambda x^{\lambda-2}+\ldots+\lambda(\lambda-2) \cdots 5 \cdot 3 x\right)+\lambda(\lambda-2) \cdots 5 \cdot 3 \sqrt{\frac{\pi}{2}} \operatorname{erf}\left(\frac{x}{\sqrt{2}}\right) .
$$

Writing out the terms of the Laguerre polynomials through [25]

$$
L_{n}^{\alpha}(x)=\sum_{m=0}^{n}(-1)^{m}\left(\begin{array}{c}
n+\alpha \\
n-m
\end{array}\right) \frac{x^{m}}{m !}=\sum_{m=0}^{n}(-1)^{m} \frac{\Gamma(n+\alpha+1)}{\Gamma(n-m+1) \Gamma(m+\alpha+1)} \frac{x^{m}}{m !},
$$

we get

$$
\begin{aligned}
\varepsilon L_{n}^{\bar{\alpha}}\left(x^{2}\right) x^{\bar{\beta}} \exp \left(-\frac{x^{2}}{2}\right)= & \int_{-\infty}^{\infty} d y \varepsilon(x-y) L_{n}^{\bar{\alpha}}\left(y^{2}\right) y^{\bar{\beta}} e^{-\frac{y^{2}}{2}} \\
& =\sum_{i=0}^{n} a_{i} \int_{-\infty}^{\infty} d y \varepsilon(x-y) y^{2 i+\bar{\beta}-1}\left(y e^{-\frac{y^{2}}{2}}\right) \\
& =a_{0} \int_{-\infty}^{\infty} d y \varepsilon(x-y) y^{\bar{\beta}-1}\left(y e^{-\frac{y^{2}}{2}}\right)+a_{1} \int_{-\infty}^{\infty} d y \varepsilon(x-y) y^{\bar{\beta}+1}\left(y e^{-\frac{y^{2}}{2}}\right)+ \\
& \ldots+a_{n} \int_{-\infty}^{\infty} d y \varepsilon(x-y) y^{2 n+\bar{\beta}-1}\left(y e^{-\frac{y^{2}}{2}}\right)
\end{aligned}
$$

where $a_{i}$ are the coefficients to $x^{i}$ in (137).

Assuming that $\bar{\beta}$ is odd, all powers in (138) are even and we use (135) on each term to give us

$$
\begin{gathered}
\varepsilon \begin{array}{l}
L_{n}^{\bar{\alpha}}\left(x^{2}\right) x^{\bar{\beta}} \exp \left(-\frac{x^{2}}{2}\right) \\
=-e^{-\frac{x^{2}}{2}}\left[a_{0}\left(x^{\bar{\beta}-1}+(\bar{\beta}-1) x^{\bar{\beta}-3}+\ldots+(\bar{\beta}-1)(\bar{\beta}-3) \cdots 4 \cdot 2\right)+\right. \\
\quad a_{1}\left(x^{\bar{\beta}+1}+(\bar{\beta}+1) x^{\bar{\beta}-1}+\ldots+(\bar{\beta}+1)(\bar{\beta}-1) \cdots 4 \cdot 2\right)+\cdots \ldots \\
\left.+a_{n}\left(x^{\bar{\beta}+2 n-1}+(\bar{\beta}+2 n-1) x^{\bar{\beta}-3}+\ldots+(\bar{\beta}+2 n-1)(\bar{\beta}+2 n-3) \cdots 4 \cdot 2\right)\right] \\
=-e^{-\frac{x^{2}}{2}}\left[\left\{a_{0}+(\bar{\beta}+1) a_{1}+(\bar{\beta}+3)(\bar{\beta}+1) a_{2}+\ldots+(\bar{\beta}+2 n-1)(\bar{\beta}+2 n-3) \cdots(\bar{\beta}+1) a_{n}\right\} \times\right. \\
\quad\left(x^{\bar{\beta}-1}+(\bar{\beta}-1) x^{\bar{\beta}-3}+(\bar{\beta}-1)(\bar{\beta}-3) x^{\bar{\beta}-5}+\cdots+(\bar{\beta}-1)(\bar{\beta}-3) \cdots 4 \cdot 2\right) \\
+x^{\bar{\beta}+1}\left(a_{1}+(\bar{\beta}+3) a_{2}+(\bar{\beta}+5)(\bar{\beta}+3) a_{3}+\ldots+(\bar{\beta}+2 n-1)(\bar{\beta}+2 n-3) \cdots(\bar{\beta}+3) a_{n}\right)+ \\
x^{\bar{\beta}+3}\left(a_{2}+(\bar{\beta}+5) a_{3}+\ldots+(\bar{\beta}+2 n-1) \cdots(\bar{\beta}+5) a_{n}\right)+\ldots \\
\left.\ldots+x^{\bar{\beta}+2 n-1} a_{n}\right] .
\end{array}
\end{gathered}
$$


When $\bar{\beta}$ is even we use (136) in (138), and we get

$$
\begin{aligned}
\varepsilon & L_{n}^{\bar{\alpha}}\left(x^{2}\right) x^{\bar{\beta}} \exp \left(-\frac{x^{2}}{2}\right) \\
=-e^{-\frac{x^{2}}{2}} & {\left[a_{0}\left(x^{\bar{\beta}-1}+(\bar{\beta}-1) x^{\bar{\beta}-3}+\ldots+(\bar{\beta}-1)(\bar{\beta}-3) \cdots 5 \cdot 3 \operatorname{erf}\left(\frac{x}{\sqrt{2}}\right)\right)+\right.} \\
& a_{1}\left(x^{\bar{\beta}+1}+(\bar{\beta}+1) x^{\bar{\beta}-1}+\ldots+(\bar{\beta}+1)(\bar{\beta}-1) \cdots 5 \cdot 3 \operatorname{erf}\left(\frac{x}{\sqrt{2}}\right)+\cdots \cdots\right. \\
+ & a_{n}\left(x^{\bar{\beta}+2 n-1}+(\bar{\beta}+2 n-1) x^{\bar{\beta}-3}+\ldots+(\bar{\beta}+2 n-1)(\bar{\beta}+2 n-3) \cdots 5 \cdot 3 \operatorname{erf}\left(\frac{x}{\sqrt{2}}\right)\right] .
\end{aligned}
$$

Using the definitions [28]

$$
\begin{gathered}
(2 k) ! ! \equiv 2 k(2 k-2) \cdots 4 \cdot 2=2^{k} \Gamma(k+1), \\
(2 k-1) ! ! \equiv(2 k-1)(2 k-3) \cdots 3 \cdot 1=\pi^{-\frac{1}{2}} 2^{k} \Gamma\left(k+\frac{1}{2}\right),
\end{gathered}
$$

where $k$ is a positive integer, we can write $(139)$ and $(140)$ in a more compact way.

For every $a_{i}, 0 \leq i \leq n$, the righthand side of eq. (139) is equal to $-e^{-x^{2} / 2}$ times a polynomial of order $(\bar{\beta}+2 i-1)$. This polynomial can be rewritten as

$$
a_{i} \sum_{j=0}^{\frac{\bar{\beta}-1}{2}+i} x^{\bar{\beta}-1-2 j+2 i} 2^{j} \frac{\Gamma\left(\frac{\bar{\beta}+1}{2}+i\right)}{\Gamma\left(\frac{\bar{\beta}+1}{2}+i-j\right)} .
$$

A similar expression exist for the polynomial in the framed part of (140). Using these expressions for the polynomial parts of (140) and (139) and inserting the Laguerre coefficients $a_{i}$ of $x^{i}$ in (137) in (140) and (139) leads to

$$
\begin{aligned}
& \mathcal{E}_{[\bar{\alpha}, \bar{\beta}, n]}(x)=\varepsilon L_{n}^{\bar{\alpha}}\left(x^{2}\right) x^{\bar{\beta}} \exp \left(-\frac{x^{2}}{2}\right)= \\
& -e^{\frac{-x^{2}}{2}}\left[\sum_{i=0}^{n} \frac{(-1)^{i}}{\Gamma(i+1)} \frac{\Gamma(n+1+\bar{\alpha})}{\Gamma(n+1-i) \Gamma(\bar{\alpha}+1+i)} \sum_{j=0}^{\frac{\bar{\beta}-1}{2}+i\left\{-\frac{3}{2}\right\}} x^{(\bar{\beta}-1-2 j+2 i)} 2^{j} \frac{\Gamma\left(\frac{\bar{\beta}+1}{2}+i\right)}{\Gamma\left(\frac{\bar{\beta}+1}{2}+i-j\right)}\right] \\
& \left\{+\sum_{i=0}^{n} \frac{(-1)^{i}}{\Gamma(i+1)} \frac{\Gamma(n+1+\bar{\alpha})}{\Gamma(n+1-i) \Gamma(\bar{\alpha}+1+i)} 2^{\left(i+\frac{\bar{\beta}}{2}\right)} \Gamma\left(i+\frac{\bar{\beta}+1}{2}\right)\left[-e^{-\frac{x^{2}}{2}} \frac{x}{\sqrt{\pi}}+\sqrt{\frac{1}{2}} \operatorname{erf}\left(\frac{x}{\sqrt{2}}\right)\right]\right\} .
\end{aligned}
$$

For even $\bar{\beta}$ we must include the terms in the brackets $\{\ldots\}$, while these are neglected for odd $\bar{\beta}$. For $\bar{\beta}=0$ a modification of the solution with the brackets is valid : for $i=0$ we must delete the term $-x e^{-x^{2} / 2} / \sqrt{\pi}$. All contributions from a negative upper limit in the summation are set to zero (for instance for $\bar{\beta}=2$ and $i=0$ the term involving $\sum_{j=0}^{-1}$ equals zero). 
B.2 $\int_{-\infty}^{\infty} d x L_{m}^{\alpha}\left(x^{2}\right) x^{\beta} e^{-\frac{x^{2}}{2}} \varepsilon L_{n}^{\bar{\alpha}}\left(x^{2}\right) x^{\bar{\beta}} e^{-\frac{x^{2}}{2}}$

Using (144) we are now able to calculate (130). Since $\beta, \bar{\beta}$ are integers, the interior and exterior functions in (130)

$$
f_{\text {int }}(x) \equiv L_{n}^{\bar{\alpha}}\left(x^{2}\right) x^{\bar{\beta}} e^{-\frac{x^{2}}{2}}
$$

and

$$
f_{\text {ext }}(x) \equiv L_{m}^{\alpha}\left(x^{2}\right) x^{\beta} e^{-\frac{x^{2}}{2}}
$$

are either even or odd. In this case it is easily shown that $\mathcal{B}_{i j}$ is non-zero only when $\bar{\beta}$ is even and $\beta$ is odd or vice visa. In the following we assumed that $\beta$ and $\bar{\beta}$ fulfills this. Using the relation $\mathcal{B}_{i j}=-\mathcal{B}_{j i}$ we can make sure that the function in the interior integral in (130) is odd. If $\bar{\beta}$ is odd this is automatically the case and we have

$$
\mathcal{B}_{i j}=\int_{-\infty}^{\infty} d x f_{\text {ext }}(x) \varepsilon f_{\text {int }}(x)
$$

with $\varepsilon f_{\text {int }}(y)$ given by (144). In case of $\bar{\beta}$ even we simply use $\mathcal{B}_{i j}=-\mathcal{B}_{j i}$ to switch the two functions

$$
\mathcal{B}_{i j}=-\mathcal{B}_{j i}=-\int_{-\infty}^{\infty} d x f_{\text {int }}(x) \varepsilon f_{\text {ext }}(x)
$$

where now $\varepsilon f_{\text {ext }}(x)$ is given by (144).

So from now on we assume $\bar{\beta}$ is odd and $\beta$ even.

Denoting by $p_{\bar{\beta}-1+2 n}$ the $(\bar{\beta}-1+2 n)$ order polynomial from (144), we want to calculate

$$
\mathcal{B}_{i j}=\int_{-\infty}^{\infty} d x L_{m}^{\alpha}\left(x^{2}\right) x^{\beta} e^{-x^{2}} p_{\bar{\beta}-1+2 n}(x)
$$

Since both $p_{\bar{\beta}-1+2 n}$ and $L_{m}^{\alpha}\left(x^{2}\right) x^{\beta} e^{-\frac{x^{2}}{2}}$ are even, we have

$$
\mathcal{B}_{i j}=-2 \int_{0}^{\infty} d x L_{m}^{\alpha}\left(x^{2}\right) x^{\beta} e^{-x^{2}} p_{\frac{\bar{\beta}-1+2 n}{2}}\left(x^{2}\right)
$$

Making the substitution $z=x^{2}$, gives

$$
\mathcal{B}_{i j}=-\int_{0}^{\infty} d z L_{m}^{\alpha}(z) z^{\frac{\beta-1}{2}} e^{-z} p_{\bar{\beta}-1+2 n}\left(z^{\frac{1}{2}}\right)
$$

Let us rewrite $p_{\bar{\beta}-1+2 n}$ using (139)

$$
\begin{aligned}
p_{\bar{\beta}-1+2 n}\left(z^{\frac{1}{2}}\right)= & b_{\bar{\beta}+2 n-1} z^{\frac{\bar{\beta}+2 n-1}{2}}+b_{(\bar{\beta}+2 n-3)} z^{\frac{\bar{\beta}+2 n-3}{2}}+\ldots+b_{(\bar{\beta}+3)} z^{\frac{\bar{\beta}+3}{2}}+b_{(\beta \overline{+} 1)} z^{\frac{\bar{\beta}+1}{2}}+ \\
& T\left(z^{\frac{\bar{\beta}-1}{2}}+b_{(\bar{\beta}-3)} z^{\frac{\bar{\beta}-3}{2}}+b_{(\bar{\beta}-5)} z^{\frac{\bar{\beta}-5}{2}}+\ldots+b_{0}\right)
\end{aligned}
$$

The coefficients $b_{i}$ are given by products of $\bar{\beta}$ and $a_{i}$, and $T$ is the factor in brackets $(\{\ldots\})$ in $(139)$. Using (141) and (142) $T$ can be reduced to :

$$
\begin{aligned}
T & =\left\{a_{0}+(\bar{\beta}+1) a_{1}+(\bar{\beta}+3)(\bar{\beta}+1) a_{2}+\ldots+(\bar{\beta}+2 n-1)(\bar{\beta}+2 n-3) \cdots(\bar{\beta}+1) a_{n}\right\} \\
& =\sum_{i=0}^{n}(-1)^{i} 2^{i} \frac{\Gamma\left(\frac{\bar{\beta}+1}{2}+i\right)}{\Gamma\left(\frac{\bar{\beta}+1}{2}\right) \Gamma(i+1)} \frac{\Gamma(n+1+\bar{\alpha})}{\Gamma(n+1-i) \Gamma(\bar{\alpha}+1+i)} .
\end{aligned}
$$


By insertion of (150) in (149) we get

$$
\begin{array}{r}
\mathcal{B}_{i j}=-\int_{0}^{\infty} d z L_{m}^{\alpha}(z) e^{-z} z^{\frac{\bar{\beta}+\beta}{2}}\left[b_{(\bar{\beta}+2 n-1)} z^{n-1}+b_{(\bar{\beta}+2 n-3)} z^{n-2}+\ldots+b_{(\bar{\beta}+3)} z+b_{(\bar{\beta}+1)}\right] \\
-T \int_{0}^{\infty} d z L_{m}^{\alpha}(z) e^{-z} z^{\frac{\bar{\beta}-1}{2}}\left(z^{\frac{(\bar{\beta}-1)}{2}}+b_{(\bar{\beta}-3)} z^{\frac{\bar{\beta}-3}{2}}+b_{(\bar{\beta}-5)} z^{\frac{\bar{\beta}-5}{2}}+\ldots+b_{0}\right) .
\end{array}
$$

We restrict the parameters to the following four cases

$$
\begin{gathered}
\alpha=\frac{\bar{\beta}+\beta}{2}, \quad \text { and } \quad(n-1)<m \quad \text { or } \quad(n-1)=m, \\
\alpha=\frac{\bar{\beta}+\beta}{2}+1, \quad \text { and } \quad(n-1)<m \quad \text { or }(n-1)=m .
\end{gathered}
$$

In case (153), orthonormality reduces (152) to

$$
\mathcal{B}_{i j}=-T \int_{0}^{\infty} d z L_{m}^{\alpha}(z) e^{-z} z^{\frac{\bar{\beta}-1}{2}}\left(z^{\frac{\bar{\beta}-1}{2}}+b_{(\bar{\beta}-3)} z^{\frac{\bar{\beta}-3}{2}}+b_{(\bar{\beta}-5)} z^{\frac{\bar{\beta}-5}{2}}+\ldots+b_{0}\right),
$$

for $(n-1)<m$, while for $(n-1)=m$ we have

$$
\begin{aligned}
\mathcal{B}_{i j}=-b_{(\bar{\beta}+2 n-1)} \int_{0}^{\infty} d z L_{m}^{\alpha}(z) e^{-z} z^{\alpha} z^{m}- \\
T \int_{0}^{\infty} d z L_{m}^{\alpha}(z) e^{-z} z^{\alpha}\left(z^{\frac{\bar{\beta}-1}{2}}+b_{(\bar{\beta}-3)} z^{\frac{\bar{\beta}-3}{2}}+b_{(\bar{\beta}-5)} z^{\frac{\bar{\beta}-5}{2}}+\ldots+b_{0}\right) .
\end{aligned}
$$

The first term in (155) reduces to

$$
\begin{array}{r}
-b_{(\bar{\beta}+2 n-1)} \int_{0}^{\infty} d z L_{m}^{\alpha}(z) e^{-z} z^{\alpha} z^{m}=-b_{(\bar{\beta}+2 n-1)} \int_{0}^{\infty} d z L_{m}^{\alpha}(z) e^{-z} z^{\alpha}\left(\frac{m !}{(-1)^{m}} L_{m}^{\alpha}(z)\right) \\
=-b_{(\bar{\beta}+2 n-1)} \frac{m !}{(-1)^{m}} h_{m}^{\alpha}=-b_{(\bar{\beta}+2 n-1)} \frac{m !}{(-1)^{m}} \frac{\Gamma(\alpha+1+m)}{m !} \\
=-b_{(\bar{\beta}+2 n-1)}(-1)^{m} \Gamma(\alpha+1+m)=-a_{n}(-1)^{m} \Gamma(\alpha+1+m) \\
=-\frac{(-1)^{n}}{n !}(-1)^{m} \Gamma(\alpha+1+m)=-\frac{(-1)^{m+1}}{(m+1) !}(-1)^{m} \Gamma(\alpha+1+m)=\frac{\Gamma(\alpha+m+1)}{\Gamma(m+2)} .
\end{array}
$$

Working out explicitly what the $b_{i}$ 's are in terms of $\Gamma$-functions and using [28]

$$
\int_{0}^{\infty} d x e^{-x} x^{\gamma-1} L_{n}^{\mu}(x)=\frac{\Gamma[\gamma] \Gamma[1+\mu+n-\gamma]}{\Gamma[n+1] \Gamma[1+\mu-\gamma]} .
$$

on each term in (155) gives us the result 


$$
\begin{aligned}
\mathcal{B}_{i j}=-\sum_{i=0}^{n}(-1)^{i} 2^{i} & \frac{\Gamma\left(\frac{\bar{\beta}+1}{2}+i\right)}{\Gamma\left(\frac{\bar{\beta}+1}{2}\right) \Gamma(i+1)} \frac{\Gamma(n+1+\bar{\alpha})}{\Gamma(n+1-i) \Gamma(\bar{\alpha}+1+i)} \times \\
& \frac{1}{\Gamma(m+1)} \sum_{j=0}^{\frac{\beta}{2}} \frac{\Gamma(\alpha-j) \Gamma(1+m+j)}{\Gamma(1+j)} 2^{j} \frac{\Gamma\left(\frac{\bar{\beta}+1}{2}\right)}{\Gamma\left(\frac{\bar{\beta}+1}{2}-j\right)}(+) \frac{\Gamma(\alpha+m+1)}{\Gamma(m+2)},
\end{aligned}
$$

for

$$
\frac{(\bar{\beta}+\beta)}{2}=\alpha, \quad(n-1) \leq m, \text { and } \bar{\beta} \text { odd },
$$

The last term is only added in the case $(n-1)=m$.

In the case $(154)$, only one term in the first line of $(152)$ survives because of orthonormality. It reads

$$
\begin{aligned}
-b_{(\bar{\beta}+1)} \int_{0}^{\infty} d z L_{m}^{\alpha}(z) e^{-z} z^{\frac{\bar{\beta}+\beta}{2}} & =-b_{(\bar{\beta}+1)} \int_{0}^{\infty} d z L_{m}^{\alpha}(z) e^{-z} z^{\frac{\bar{\beta}+\beta}{2}+1} z^{-1} \\
& =-b_{(\bar{\beta}+1)} \int_{0}^{\infty} d z L_{m}^{\alpha}(z) e^{-z} z^{\alpha} z^{-1}=-b_{(\bar{\beta}+1)} \Gamma(\alpha) .
\end{aligned}
$$

By $(139)$ and (150) we have

$$
\begin{aligned}
b_{(\bar{\beta}+1)} & =\left(a_{1}+(\bar{\beta}+3) a_{2}+(\bar{\beta}+5)(\bar{\beta}+3) a_{3}+\ldots+(\bar{\beta}+2 n-1)(\bar{\beta}+2 n-3) \cdots(\bar{\beta}+3) a_{n} \cdot\right) \\
& =\frac{T-a_{0}}{(\bar{\beta}+1)} .
\end{aligned}
$$

The contribution from the second line in $(152)$ is given by $(159)$ without the $(+)$ term and with the substitution $\alpha \rightarrow \alpha-1$. Collecting the parts we get that for

$$
\frac{\bar{\beta}+\beta}{2}+1=\alpha, \quad(n-1) \leq m \quad \text {, and } \quad \bar{\beta} \text { odd }
$$

we have

$$
\mathcal{B}_{i j}=[\text { Righthand side of eq. (159) with } \alpha \rightarrow \alpha-1 \text { and without the term }(+)]+\frac{T-a_{0}}{\bar{\beta}+1} \Gamma(\alpha),
$$

where $T$ is given by eq. (151).

\section{References}

[1] E.V.Shuryak and J.J.M.Verbaarschot, Nucl.Phys. A560 (1993) 306.

[2] M.A.Halasz and J.J.M.Verbaarschot, Phys.Rev. D52 (1995) 2563. 
[3] A.Smilga and J.J.M.Verbaarschot, Phys.Rev. D51 (1995) 829.

[4] J.J.M.Verbaarschot and I.Zahed, Phys.Rev.Lett. 73 (1994) 2288.

[5] J.Gasser and H.Leutwyler, Phys.Lett. B184 (1987) 83.

[6] T.Banks and A.Casher, Nucl.Phys. B169 (1980) 103.

[7] H.Leutwyler and A.Smilga, Phys.Rev. D46 (1992) 5607.

[8] J.J.M.Verbaarschot and I.Zahed, Phys.Rev.Lett. 70 (1993) 3852.

[9] J.J.M.Verbaarschot, Phys.Rev.Lett. 72 (1994) 2531, Nucl.Phys. B426 (1994) 559.

[10] U.Magnea: The ortogonal ensemble of random matrices and $Q C D$ in three dimensions, hepth/9907096.

[11] U.Magnea: Three-dimensional $Q C D$ in the adjoint representation and random matrix theory, hep-th/9912207.

[12] R.D.Pisarski, Phys.Rev. D29 (1984) 2423.

[13] G.Akemann, P.H.Damgaard, U.Magnea and S.Nishigaki, Nucl.Phys. B487 (1997) 721.

[14] M.K.Sener and J.J.M.Verbaarschot, Phys.Rev.Lett. 81 (1998) 248.

[15] J.J.M.Verbaarschot : Universal Behavior in Dirac Spectra, hep-th/9710114.

[16] J.Christiansen, Nucl.Phys. B547 (1998) 329.

[17] P.H.Damgaard and S.Nishigaki, Phys.Rev. D57 (1998) 5299.

[18] M. L. Mehta: Random Matrices, 2nd Edition, Academic Press (1991).

[19] H. Widom, J.Statist.Phys. 94 (1999) 347.

[20] T.Nagao and K.Slevin, J.Math. 34 (1993) 2075.

[21] G. Szego : Orthogonal Polynomials, 4th ed. Am. Math. Soc.,Providence, 1975.

[22] F.J.Dyson, Comm.Math. 19 (1970) 235.

[23] G.Mahoux and M.L Mehta, J.Phys.I. (France) 1 (1991) 1093.

[24] C.A.Tracy and H. Widom : Correlation Functions, Cluster Functions and Spacing Distribibutions for Random Matrices, solv-int/9804004.

[25] W.Magnus, F.Oberhettinger and R.P.Soni : Formulars and Theorems for the Special Functions of Mathematical Physics, 3.Edition, Springer-Verlag Berlin Heidelberg New York 1966.

[26] C.A.Craig and H. Widom, Commun.Math.Phys. 163 (1994) 33.

[27] J.J.M.Verbaarschot and A.Smilga, Phys.Rev. D51 (1995) 829.

[28] A.Erdélyi (ed.) et al : Higher Transcendental Functions, Vol.II, McGraw-Hill (1953).

[29] T.Nagoa and S.M.Nishigaki, hep-th/0005077. 